\title{
The impact of static and dynamic roughness elements on flow separation
}

\author{
P. Servini ${ }^{1}$, F. T. Smith ${ }^{1} \dagger$ and A. P. Rothmayer ${ }^{2}$ \\ ${ }^{1}$ Department of Mathematics, University College London, Gower Street, London WC1E 6BT, \\ UK \\ ${ }^{2}$ Department of Aerospace Engineering, Iowa State University, Ames, Iowa 50011, USA
}

(Received $\mathrm{xx}$; revised $\mathrm{xx}$; accepted $\mathrm{xx}$ )

The use of static or dynamic roughness elements has been shown in the past to delay the separation of a laminar boundary layer from a solid surface. Here, we examine analytically the effect of such elements on the local and breakaway separation points, corresponding respectively to the position of zero skin friction and presence of a singularity in the roughness region, for flow over a hump embedded within the boundary layer. Two types of roughness elements are studied: the first is small and placed near the point of vanishing skin friction; the second is larger and extends downstream. The forced flow solution is found as a sum of Fourier modes, reflecting the fixed frequency forcing of the dynamic roughness. Solutions for both the static and dynamic roughness show that the presence of the roughness element is able to move the separation points downstream, given an appropriate choice of roughness frequency, height, position and width. This choice is found to be qualitatively similar to that observed for leading-edge separation. Furthermore, for a negative static roughness a small region of separated flow forms at high roughness depth, although there is a critical depth above which boundary-layer breakaway moves suddenly upstream.

\section{Key words:}

\section{Introduction}

Flow separation is the focus of this work, especially the possible use of oscillating roughness elements to affect the separation position on an otherwise fixed solid surface. It is motivated by applications to laminar flow control and to increased understanding of bird flight, among other interests.

The search for effective laminar flow techniques to prevent separation, while avoiding turbulence and the higher skin friction drag associated with it, remains of great importance, with applications including drones, propellers, wind turbines and helicopters with chord Reynolds numbers between $10^{4}$ and $10^{6}$ (Lissaman 1983; Gad-el Hak 2000). Relatively well-established techniques include streamlining, which can reduce the adverse pressure gradient encountered or move back the position of minimum pressure, and suction (Atik et al. 2005) to remove slower, near-wall particles and entrain faster ones; while the use of wall heat transfer (Chang 1970) and rotating cylinders (Modi 1997) has also been studied. Tripping the boundary layer to go turbulent through the use of roughness elements (whether active (Tani 1969) or passive (Pruessner \& Smith 2015))

$\dagger$ Email address for correspondence: f.smith@ucl.ac.uk 
also remains popular when avoiding separation. Other ideas, for example from nature (Bushnell \& Moore 1991; Fish \& Lauder 2006), may also inspire new and novel techniques.

The use of any flow control device must be offset by the costs, both financial and in terms of introduced drag, involved in incorporating the associated equipment. Hence potential devices should be lightweight, easy to implement and require the minimal amount of energy to operate. The pioneering studies of Huebsch (2006), Rothmayer \& Huebsch (2011, 2012), Huebsch et al. (2012) and Grager et al. (2012) on dynamic roughness elements - small bumps embedded within a boundary layer and made to oscillate at a given frequency - is therefore promising. These dynamic roughness elements can be created through the use of a pressure plenum within the airfoil (as in Grager et al. (2012)) or possibly by electro-active polymers (EAPs) (Dearing et al. 2007; DeMauro et al. 2015) or microelectromechanical systems (MEMS) and have been shown, both experimentally and numerically, to increase the angle of attack at which separation occurs.

Equally inspiring is the study by Braun \& Kluwick (2004) (henceforth referred to as $\mathrm{BK}$ ), which is particularly relevant to the study of such dynamic roughness elements and concerns marginal separation. The local separation of a steady two-dimensional flow from the surface of a body is generally taken to occur where the skin friction becomes zero after a length of attached flow (positive skin friction) immediately upstream. At this point, the original surface streamline leaves the surface and carries with it the original boundary layer in a sense, possibly leading to a larger breakaway separation process with a region of relatively slow recirculating fluid existing beneath the dividing streamline. The point of vanishing skin friction also heralds the breakdown of classical boundary layer theory (Prandtl 1904), with the appearance either of Goldstein's square root singularity (Goldstein 1948) or the weaker singularity that accompanies marginal separation (Ruban 1981; Stewartson et al. 1982). In the latter case, and within limits, the skin friction goes through zero but then immediately recovers: this phenomenon has been shown to occur in flows over slender airfoils at low angles of attack (Ruban 1982) or smooth backwardfacing steps (Schlichting \& Gersten 2000); channel flows with suction (Hsiao \& Pauley 1994); or a viscous wall jet that is made to deflect (Zametaev 1986). For all of these, marginal separation occurs only if the value of some parameter governing the flow is below a critical threshold.

In aerodynamics, this parameter is linked to the angle of attack of the airfoil and thereby to the adverse pressure gradient encountered on the suction side. As the flow proceeds from the stagnation point at the front of the airfoil, the pressure first reaches a minimum near the leading edge, before recovering. This increase in pressure results in near-wall particles slowing down and eventually being moved to some finite distance from the surface by the reversed flow that exists once the skin friction has become negative. For thin airfoils, where the Reynolds number (based on the radius of curvature of the leading edge) is comparatively low, the flow tends to remain laminar and thus is more likely to separate: as the angle of attack increases, marginally separated flow is initially encountered, with the accompanying creation of a short bubble typically no more than $1 \%$ of the length of the airfoil chord. This situation exists up to some critical value $\Gamma_{c}$ of the increment $\Gamma$ of the angle of attack, beyond which no feasible solutions to the marginal separation equations exist, indicating the 'bursting' of the bubble into either a longer one or a fully developed region of separated flow. This results in a severe loss of lift and dramatic increase in drag on the airfoil; with similar detrimental effects occurring also for internal flows (Sychev et al. 1998).

In detail, BK considered an airfoil on which an unsteady, three-dimensional object was mounted, in order to explore what happened in marginally separated flow at or 
near the critical value of the angle of attack increment, which they represent by $\Gamma_{c}$. To maintain consistency between this paper and those of BK (Braun \& Kluwick 2002, 2004), we will keep the same notation. A bifurcation takes place for subcritical values of $\Gamma$, with two flow regimes possible (Braun \& Kluwick 2002); while for supercritical values, as mentioned, no steady solution is possible. The object represents a steady roughness shape $\left(h_{s}\right)$ with small time-dependent variations $\left(h_{u}\right)$ of this shape. Of interest for flow control applications is that $h_{s}$ allows one to increase the critical value $\Gamma_{c}$, suggesting that a greater angle of attack can be achieved before the flow forms either a large separation bubble or fully separates and stalls. The higher order unsteady contributions are used to analyse the bubble-bursting phenomenon.

The main question in the current work is whether such positive effects can occur in other significant configurations and specifically in the near-wall motion over a small hump within a boundary layer or channel flow at high Reynolds number. The latter 'condensed flow' is described by Smith \& Daniels (1981) (SD) in the context of the removal of the Goldstein singularity at separation. Although not strictly in the realm of marginal separation (there is no critical parameter here), we focus on the local and breakaway separation positions and ask whether a tiny roughness element, either static or dynamic, is able to shift them downstream and, if so, how position, length, height and oscillation frequency affect the shift. Loosely equating the increase in $\Gamma_{c}$ with a downstream shift in the separation position, we find that the dependence of both on the aforementioned parameters is qualitatively similar. In contrast to the work of BK, for our dynamic roughness we take the oscillation amplitude of the roughness element to be of the same order as the roughness height, thereby considering a positive roughness to drop flush to the surface before increasing to its maximum extension within one cycle of oscillation. We will also answer the question of whether the dynamic roughness can move the separation point further downstream as compared to the steady roughness, once the average over a period of oscillation is taken.

Section 2 below describes the model with the various regions of SD, deriving the governing equations in the region of vanishing skin friction where the roughness element is active, i.e. the 'roughness region'. In $\S \S 3,4$ we present the results for a static and dynamic roughness impacting on the local separation point; in section $\S 5$, we consider the effect of larger roughness elements on breakaway separation, with the form of the pressuredisplacement equation derived in $§ 2.2$ suggesting the possibility of introducing favourable pressure perturbations to the flow; and conclusions and scope for future research close in $\S 6$.

\section{The model}

Our planar flow is incompressible and the boundary layer, lying on the wall $y^{*}=0$ with classical thickness $O\left(R e^{-1 / 2}\right)$, is laminar. The Reynolds number $(R e)$, taken to be large, is the ratio of inertial to viscous forces: $R e=U_{\infty} L / \nu$, where $U_{\infty}$ is the speed of the oncoming flow, $L$ is a characteristic length scale and $\nu$ is the kinematic viscosity. Asterisked variables are non-dimensional, with dimensional equivalents given by $L\left(x^{*}, y^{*}\right), U_{\infty}\left(u^{*}, v^{*}\right), L t^{*} / U_{\infty}$ and $\rho U_{\infty}^{2} p^{*}$, where $\rho$ is the fluid density. As in SD, the flow encounters a hump, given by

$$
y^{*}=R e^{-1 / 2} \delta\left[h F\left(x^{*}\right)+\hat{f}\left(h, x^{*}, t^{*}\right)\right],
$$

completely embedded within the classical boundary layer, with $R e^{-1 / 2} \delta$ the characteristic hump height (which, with $\delta \ll 1$, is much smaller than the boundary layer thickness) 
and $h$ the non-dimensional height factor of the bump. Here, $F$ is the hump shape, which achieves a maximum at $x^{*}=x_{\max }^{*}$, after which the pressure gradient becomes adverse and drives the flow towards separation. The additional contribution $\hat{f}$ corresponds to the roughness element, which is introduced in the region where the skin friction vanishes (see $§ 2.2$ ) but is identically zero everywhere else. Its crucial height scale, which is some power of $h$, will be determined later. Finally, the streamwise coordinate $x^{*}$ and time $t^{*}$ are based on the non-dimensional length scale of the bump, which in turn is based on the Reynolds number as shown in equation (2.1a).

As mentioned in the introduction, the local separation point coincides with the position of zero skin friction and the appearance of the Goldstein singularity but, as demonstrated by $\mathrm{SD}$, this singularity is moved downstream and eventually removed completely. This is through physical interactions in different flow regions (see below), and is seen most readily when the characteristic length $(\ell)$ and height scales of the hump satisfy $R e^{-3 / 4} \ll$ $\ell \ll R e^{-3 / 8}$ and $R e^{-1 / 4} \ll \delta \ll R e^{-1 / 8}$ (Smith et al. 1981). Then the coordinates and variables scale as

$$
\begin{aligned}
& x^{*}=\ell x, \quad y^{*}=R e^{-\frac{1}{2}} \ell^{\frac{1}{3}}(y+h F+\hat{f}), \quad t^{*}=\ell^{\frac{2}{3}} t, \\
& p^{*}=\ell^{\frac{2}{3}} p, \quad u^{*}=\ell^{\frac{1}{3}} u, \quad v^{*}=R e^{-\frac{1}{2}} \ell^{-\frac{1}{3}}\left[v+\frac{\partial \hat{f}}{\partial t}+u \frac{\partial}{\partial x}(h F+\hat{f})\right],
\end{aligned}
$$

with all unasterisked quantities of $O(1)$, where a Prandtl transposition has been applied to simplify the no-slip condition of equation (2.3). Substituting into the Navier-Stokes equations, we obtain, written in terms of the stream function $\psi$, the unsteady condensedflow equations

$$
u=\frac{\partial \psi}{\partial y}, \quad v=-\frac{\partial \psi}{\partial x}, \quad \frac{\partial^{2} \psi}{\partial y \partial t}+\frac{\partial \psi}{\partial y} \frac{\partial^{2} \psi}{\partial x \partial y}-\frac{\partial \psi}{\partial x} \frac{\partial^{2} \psi}{\partial y^{2}}=-\frac{\partial p}{\partial x}(x, t)+\frac{\partial^{3} \psi}{\partial y^{3}} .
$$

These are subject to the boundary conditions

$$
\begin{aligned}
& \psi=\frac{\partial \psi}{\partial y}=0 \quad \text { on } y=0 \\
& \psi \rightarrow \frac{1}{2} y^{2}, \quad p \rightarrow 0 \quad \text { as } x \rightarrow-\infty \\
& u \sim y+h F(x)+\hat{f}(h, x, t) \quad \text { as } y \rightarrow \infty,
\end{aligned}
$$

corresponding to no-slip, matching with the boundary layer far upstream and the requirement that there be no displacement of the original boundary layer and outer inviscid flow due to the presence of the (relatively) small hump. We note that this system is valid for all values of the non-dimensional hump height $(h)$ strictly between zero and infinity, provided that $h \ll \delta^{-1} R e^{-1 / 8}$ (for example, for bumps with a length scale equal to the boundary layer height, $\ell \sim R e^{-1 / 2}$, one obtains a bump height scale $\delta \sim \ell^{1 / 3} \sim R e^{-1 / 6}$; and hence $h \ll R e^{1 / 24}$ ), and we will focus here on cases where $h \gg 1$, as per the work of Smith \& Daniels (1981): small $h$, albeit without dynamic roughness, was studied by Smith (1976b), while situations where $h=O(1)$ appear in Smith (1976a).

\subsection{The flow development}

The development of the flow can be divided into the following regions: the flow upstream of the hump and over its front face is attached and can be dealt with using classical boundary layer theory (region A in figure 1); this classical approach breaks down as the Goldstein singularity is approached at the rear of the hump, and the singularity 
Boundary layer edge

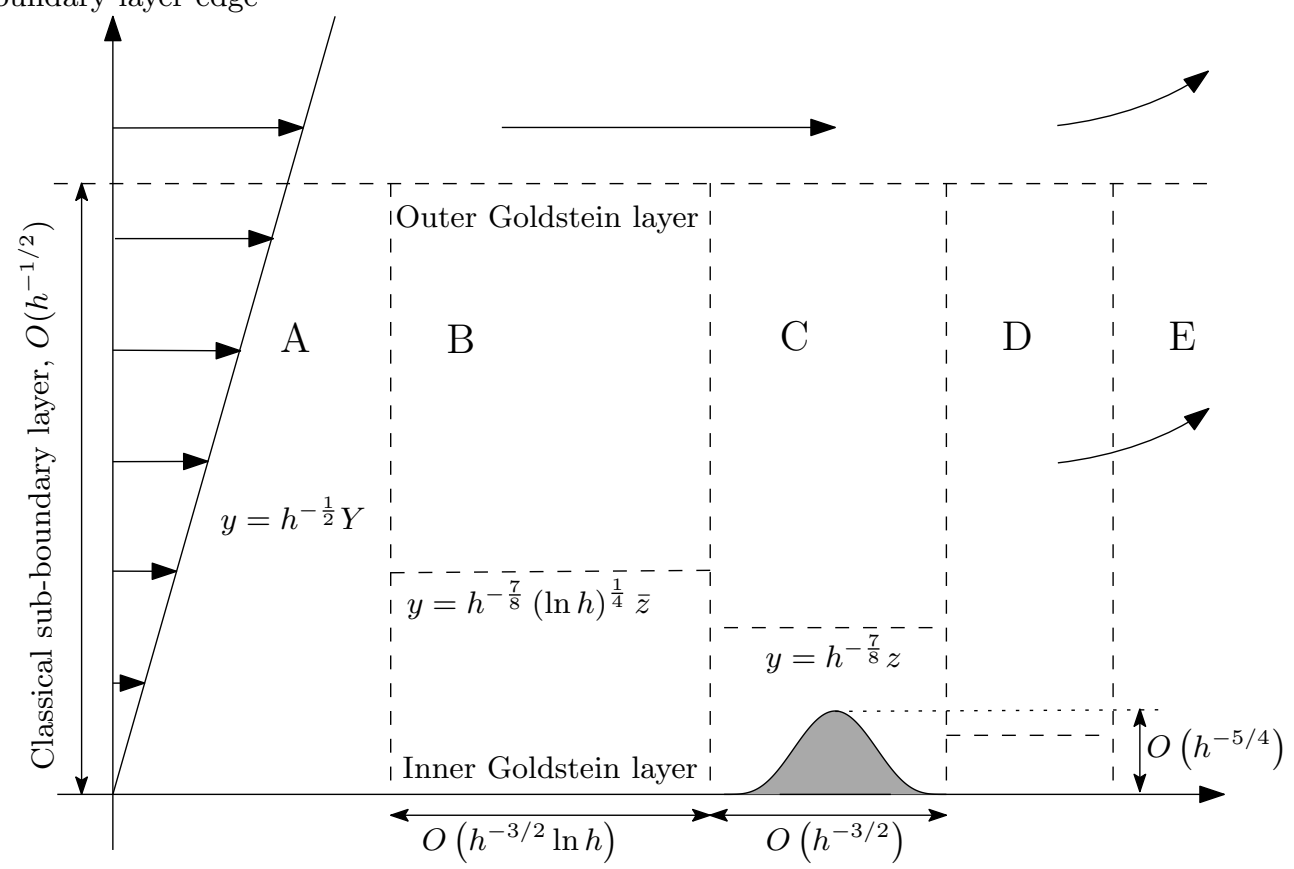

Figure 1 . The development of the flow in the $x, y$-plane as it reaches the Goldstein singularity at the end of region A and proceeds downstream. A summary of each flow region is given in the text; the dynamic roughness (grey) is placed in region C, which has a length scale of $O\left(h^{-3 / 2}\right)$. Not to scale.

can be shifted downstream by considering a smaller length scale around the singular point (region B), with the boundary layer split vertically into two sublayers; the singularity can then be removed completely by considering a still smaller length scale, allowing the skin friction to pass smoothly through zero (region $\mathrm{C}$ ); complete nonlinear breakaway of the near-wall layer, associated with the presence of a (removable) singularity in region $\mathrm{C}$, occurs in a fourth region (D); upstream of the separated flow (E) reattaching further downstream. Our interest is mainly in the positions of zero skin friction $(\S \S 3,4)$ and the removable singularity appearing downstream of it ( 55 ), both of which occur in region $\mathrm{C}$, which is where the roughness element will be placed. In this section, we summarise the solutions to the stream functions and pressures in regions $\mathrm{A}$ and $\mathrm{B}$, before focusing on region $\mathrm{C}$ in the next section. The effect of the roughness is expected to be mostly local and thus for the flow in regions D and E, we refer the reader to the SD paper.

The steady flow upstream of the Goldstein singularity consists of a viscous near-wall region of vertical scale $O\left(h^{-1 / 2}\right)$ and a large inviscid outer zone where $y=O(h)$. The boundary condition at infinity sets the form of the stream function in the outer layer; imposing the condition of no normal flow at $y=0$ in the outer layer solution gives the leading order pressure term, which drives the leading order flow in the viscous sublayer. Matching determines the next term in the pressure expansion, and so on. The outer layer stream function is then

$$
\psi=\frac{1}{2}[y+h F(x)]^{2}+p
$$


and the viscous layer stream function responds as

$$
\psi \sim h^{\frac{1}{2}} F(x)\left[Y-\beta_{0}(x)\right]+h^{-1}\left[\frac{1}{2} Y^{2}+p_{2}(x)\right]+\ldots
$$

as $Y \rightarrow \infty$, with a pressure expansion of

$$
p=-\frac{1}{2} h^{2} F^{2}(x)-h^{\frac{1}{2}} \beta_{0}(x) F(x)+\ldots .
$$

Here, $Y$ is the scaled normal coordinate in the inner layer, given by $y=h^{-1 / 2} Y$, and $\beta_{0}(x)$ is obtained from the numerical solution to the classical boundary layer equations obtained in that layer.

Denoting by $x_{\mathrm{s}}$ the position at which the scaled skin friction $\left(\tau_{\text {wall }} \equiv \partial^{2} \psi / \partial y^{2}\right.$ evaluated at $y=0$ ) vanishes, Goldstein (1948) showed that the above procedure first outlined by Prandtl gives rise to a square-root singularity as $x$ approaches $x_{\mathrm{s}}$. If we look at the limit as $x \rightarrow x_{\mathrm{s}}^{-}$, the viscous layer splits into two decks: an outer Goldstein layer, where $Y$ remains of $O(1)$; and an inner Goldstein layer, where the vertical coordinate is scaled as $\eta=Y\left(x_{\mathrm{s}}-x\right)^{-1 / 4} \sim 1$. The stream function in the inner Goldstein layer then behaves as

$$
\psi^{\mathrm{i}}=h^{\frac{1}{2}}\left[\frac{1}{6} \mu\left(x_{\mathrm{s}}-x\right)^{\frac{3}{4}} \eta^{3}+\alpha_{0}\left(x_{\mathrm{s}}-x\right) \eta^{2}+\left(x_{\mathrm{s}}-x\right)^{\frac{5}{4}}\left(\alpha_{1} \eta^{2}-\frac{1}{60} \alpha_{0}^{2} \eta^{5}\right)\right]+\ldots,
$$

from which one can identify the square-root singularity in $\tau_{\text {wall }}$. Here $\mu=-F_{\mathrm{s}} F_{\mathrm{s}}^{\prime}>0$, where $F_{\mathrm{s}}=F\left(x_{\mathrm{s}}\right)$; and $\alpha_{0}$ and $\alpha_{1} \propto \alpha_{0}^{2}$ are unknown, non-zero, positive constants. The outer Goldstein layer stream function can be represented as

$$
\psi^{\mathrm{o}}=h^{\frac{1}{2}}\left[\psi_{0 \mathrm{~s}}(Y)+\frac{2 \alpha_{0}}{\mu}\left(x_{\mathrm{s}}-x\right)^{\frac{1}{2}} \psi_{0 \mathrm{~s}}^{\prime}(Y)+\frac{2 \alpha_{1}}{\mu}\left(x_{\mathrm{s}}-x\right)^{\frac{3}{4}} \psi_{0 \mathrm{~s}}^{\prime}(Y)\right]+\ldots,
$$

where

$$
\left.\begin{array}{l}
\psi_{0 \mathrm{~s}}(Y) \sim \frac{1}{6} \mu Y^{3}-\frac{1}{60} \alpha_{0}^{2} Y^{5}+\ldots \quad \text { as } Y \rightarrow 0 \\
\psi_{0 \mathrm{~s}}(Y) \sim F_{\mathrm{s}}\left(Y-\beta_{\mathrm{s}}\right)+o(1) \quad \text { as } Y \rightarrow \infty,
\end{array}\right\}
$$

with $\beta_{\mathrm{s}}=\beta\left(x_{\mathrm{s}}\right)$ being constant.

Removal of the Goldstein singularity begins over the length scale $x-x_{\mathrm{s}} \sim h^{-3 / 2} \ln h$ of region B. The outer Goldstein layer retains the height scale $Y \sim 1$, while the inner Goldstein layer has $Y=h^{-3 / 8}(\ln h)^{1 / 4} \bar{z}$ and we introduce the new streamwise coordinate $x-x_{\mathrm{s}}=h^{-3 / 2} \ln (h) \bar{X} ; \bar{X}, \bar{z} \sim 1$. The relevant stream functions (SD) are then

$$
\begin{aligned}
\bar{\psi}^{\mathrm{o}}= & h^{\frac{1}{2}} \psi_{0 \mathrm{~s}}(Y)+\psi_{0 \mathrm{~s}}^{\prime}(Y)\left[h^{-\frac{1}{4}}(\ln h)^{\frac{1}{2}} \bar{\alpha}_{1}(\bar{X})\right. \\
& \left.+h^{-\frac{1}{4}}(\ln h)^{-\frac{1}{2}} \ln (\ln h) \bar{\alpha}_{1 L}(\bar{X})+h^{-\frac{1}{4}}(\ln h)^{-\frac{1}{2}} \bar{\alpha}_{2}(\bar{X})\right]+\ldots \\
\bar{\psi}^{\mathrm{i}}= & h^{-\frac{5}{8}}(\ln h)^{\frac{3}{4}}\left(\frac{1}{6} \mu \bar{z}^{3}\right)+\left(\frac{1}{2} \mu \bar{z}^{2}\right)\left[h^{-1}(\ln h) \bar{\alpha}_{1}(\bar{X})+h^{-1} \ln (\ln h) \bar{\alpha}_{1 L}(\bar{X})\right. \\
& \left.+h^{-1} \bar{\alpha}_{2}(\bar{X})\right]+h^{-\frac{11}{8}}(\ln h)^{\frac{5}{4}}\left(\frac{1}{2} \mu \bar{z}^{2} \bar{\alpha}_{3}(\bar{X})-\frac{1}{60} \alpha_{0}^{2} \bar{z}^{5}\right)+\ldots
\end{aligned}
$$

for the outer and inner Goldstein layers respectively, and

$$
\begin{aligned}
p= & h^{2}\left(-\frac{1}{2} F_{\mathrm{s}}^{2}\right)+h^{\frac{1}{2}} \ln h(\mu \bar{X})+h^{\frac{1}{2}}\left(-\beta_{\mathrm{s}} F_{\mathrm{s}}\right)+F_{\mathrm{s}}\left[h^{-\frac{1}{4}}(\ln h)^{\frac{1}{2}} \bar{\alpha}_{1}(\bar{X})\right. \\
& \left.+h^{-\frac{1}{4}}(\ln h)^{-\frac{1}{2}} \ln (\ln h) \bar{\alpha}_{1 L}(\bar{X})+h^{-\frac{1}{4}}(\ln h)^{-\frac{1}{2}} \bar{\alpha}_{2}(\bar{X})\right]+\ldots
\end{aligned}
$$


for the pressure; also

$$
\begin{aligned}
\bar{\alpha}_{1}(\bar{X})= & 2 \mu^{-1} \alpha_{0}\left(-\bar{X}+\frac{3 A_{0 L}}{2 \alpha_{0}}\right)^{\frac{1}{2}}, \\
\bar{\alpha}_{1 L}(\bar{X})= & -\mu^{-1} A_{0 L}\left(-\bar{X}+\frac{3 A_{0 L}}{2 \alpha_{0}}\right)^{-\frac{1}{2}}, \\
\bar{\alpha}_{2}(\bar{X})= & -\mu^{-1} A_{0 L}\left(-\bar{X}+\frac{3 A_{0 L}}{2 \alpha_{0}}\right)^{-\frac{1}{2}} \ln \left(-\bar{X}+\frac{3 A_{0 L}}{2 \alpha_{0}}\right) \\
& +\mu^{-1} A_{0}\left(-\bar{X}+\frac{3 A_{0 L}}{2 \alpha_{0}}\right)^{-\frac{1}{2}}, \\
A_{0 L}= & \frac{F_{\mathrm{s}}\left(-\frac{1}{4}\right) !}{2^{\frac{5}{2}} \mu^{\frac{1}{2}}\left(\frac{1}{4}\right) !}
\end{aligned}
$$

and $\bar{\alpha}_{3}, A_{0}$ are associated functions and constants.

The above expansions result in a scaled skin friction of

$$
\tau_{\text {wall }}=2 \alpha_{0} h^{\frac{3}{4}}(\ln h)^{\frac{1}{2}}\left(-\bar{X}+\frac{3 A_{0 L}}{2 \alpha_{0}}\right)^{\frac{1}{2}}+\ldots,
$$

indicating that the Goldstein singularity has merely been shifted to the position $\bar{X}=$ $3 A_{0 L} / 2 \alpha_{0}$. Its complete removal, as well as the introduction of the dynamic roughness, is considered in the following section.

\subsection{The roughness region}

As $\bar{X}$ approaches $3 A_{0 L} / 2 \alpha_{0}$, the stream function expansion in the lower Goldstein layer (2.10) breaks down. In particular, this occurs when

$$
\left(-\bar{X}+\frac{3 A_{0 L}}{2 \alpha_{0}}\right) \sim(\ln h)^{-1} .
$$

We therefore define coordinates $(X, z)$ in the inner and $(X, Y)$ in the outer Goldstein layers, all of $O(1)$, as

$$
x-x_{\mathrm{s}}=h^{-\frac{3}{2}} \ln (h) \frac{3 A_{0 L}}{2 \alpha_{0}}+h^{-\frac{3}{2}} X, \quad y=h^{-\frac{7}{8}} z, \quad \text { and } y=h^{-\frac{1}{2}} Y .
$$

It is in this region that we introduce the roughness element in order to investigate the impact it has both on the position of zero skin friction and downstream singularity. For the purposes of the following derivation, we will take the roughness element to be timedependent, although static elements will also be studied in $\S \S 3,5$. At the former, $\tau_{\text {wall }}$ will pass regularly through zero, while the latter is also removable in a physically sensible fashion in region D and corresponds to the full nonlinear breakaway of the boundary layer (SD). The height scale of the roughness element is $O\left(h^{-5 / 4}\right)$ and the oscillation frequency is of order $h^{11 / 8}$, indicating the scaled time

$$
T=h^{11 / 8} t:
$$

these scalings affect the governing equations at the appropriate order, but other possible choices will be referred to at the end of this section.

The boundary condition at infinity, $(2.3 c)$, can be integrated with respect to $y$ to 
obtain the stream function behaviour

$$
\psi \sim \frac{1}{2}\left[y+h F(x)+h^{-\frac{5}{4}} f(x, T)\right]^{2}+q(x, T) \quad \text { as } y \rightarrow \infty,
$$

where $\hat{f}=h^{-5 / 4} f$. The function $q$ is determined by taking the limit of the condensed flow equation (2.2) as $y \rightarrow \infty$ and gives

$$
q(x, T)=p(x, T)+h^{\frac{1}{8}} \int_{-\infty}^{x} \frac{\partial f}{\partial T}(s, T) \mathrm{d} s .
$$

Thus the stream function behaves as

$$
\psi \sim \frac{1}{2}\left[y+h F(x)+h^{-\frac{5}{4}} f(x, T)\right]^{2}+p(x, T)+h^{\frac{1}{8}} \int_{-\infty}^{x} \frac{\partial f}{\partial T}(s, T) \mathrm{d} s
$$

when $y \rightarrow \infty$. Note that the above is valid throughout the streamwise extent of the hump, $x \sim 1$, under the understanding that $f$ is identically zero everywhere outside the small roughness region defined above. Thus there is no contradiction with the previous equation (2.4).

The form of the pressure in this region is derived from the upstream pressure (2.11), which is rewritten using the new streamwise coordinate $X$ to suggest the expansion

$$
p(X, T)=-h^{2}\left(\frac{1}{2} F_{\mathrm{s}}^{2}\right)+h^{\frac{1}{2}}(\ln h) \frac{3 A_{0 L}}{2 \alpha_{0}} \mu+h^{\frac{1}{2}}\left(\mu \bar{X}-\beta_{\mathrm{s}} F_{\mathrm{s}}\right)+h^{-\frac{1}{4}} P_{1}+\ldots
$$

The stream function in the outer Goldstein layer must match with that of (2.13) as $Y \rightarrow \infty$; and thus expanding the latter about $x_{\mathrm{s}}$ and rewriting it in terms of the normal coordinate $Y$, provides the expansion

$$
\Psi^{\mathrm{o}}(X, Y, T)=h^{\frac{1}{2}} \Psi_{1}^{\mathrm{o}}(X, Y, T)+h^{-\frac{1}{4}} \Psi_{2}^{\mathrm{o}}(X, Y, T)+\ldots,
$$

with the functions $\Psi_{i}^{\mathrm{o}}$ satisfying the matching conditions

$$
\begin{aligned}
& \Psi_{1}^{\mathrm{o}} \rightarrow F_{\mathrm{s}}\left(Y-\beta_{\mathrm{s}}\right) \quad \text { as } Y \rightarrow \infty, \\
& \Psi_{1}^{\mathrm{o}} \rightarrow \psi_{0 \mathrm{~s}}(Y) \text { as } X \rightarrow-\infty, \\
& \Psi_{2}^{\mathrm{o}} \rightarrow P_{1}(X, T)+F_{\mathrm{s}} f(X, T) \quad \text { as } Y \rightarrow \infty, \\
& \Psi_{2}^{\mathrm{o}} \rightarrow 2 \mu^{-1} \alpha_{0} \psi_{0 \mathrm{~s}}^{\prime}(Y)|X|^{\frac{1}{2}}+\ldots \quad \text { as } X \rightarrow-\infty ;
\end{aligned}
$$

the upstream conditions here arise from equation $(2.10 a)$.

Substitution of the expansions (2.14) and (2.15) into the condensed flow equation (2.2) gives the governing equations for the components $\Psi_{i}^{\mathrm{o}}$, solved subject to the matching conditions (2.16) above. Thus the equation

$$
\frac{\partial \Psi_{1}^{\mathrm{o}}}{\partial Y} \frac{\partial^{2} \Psi_{1}^{\mathrm{o}}}{\partial X \partial Y}-\frac{\partial \Psi_{1}^{\mathrm{o}}}{\partial X} \frac{\partial^{2} \Psi_{1}^{\mathrm{o}}}{\partial Y^{2}}=0
$$

for $\Psi_{1}^{\mathrm{o}}$ is solved to obtain

$$
\Psi_{1}^{\mathrm{o}}=\psi_{0 \mathrm{~s}}(Y)
$$

and

for

$$
\psi_{0 \mathrm{~s}}^{\prime} \frac{\partial^{2} \Psi_{2}^{\mathrm{o}}}{\partial X \partial Y}-\psi_{0 \mathrm{~s}}^{\prime \prime} \frac{\partial \Psi_{2}^{\mathrm{o}}}{\partial X}=0
$$

$$
\Psi_{2}^{\mathrm{o}}=\psi_{0 \mathrm{~s}}^{\prime}(Y)[A(X, T)+f(X, T)],
$$


using the properties of $\psi_{0 \text { s }}$ given in (2.9). Hence

$$
\Psi^{\mathrm{o}}=h^{\frac{1}{2}} \psi_{0 \mathrm{~s}}(Y)+h^{-\frac{1}{4}} \psi_{0 \mathrm{~s}}^{\prime}(Y)[A(X, T)+f(X, T)]+\ldots
$$

is the stream function to the first two orders in the upper Goldstein layer. Terms at orders $h^{-1}$ and $h^{-11 / 8}$ also affect the lower layer expansion of (2.19) below.

The displacement function $A$ is related to the pressure through the pressuredisplacement relation

$$
P_{1}=F_{\mathrm{s}} A,
$$

on applying the condition $(2.16 c)$. This displacement function must be found by considering the flow in the inner Goldstein layer and thus the outer layer causes interaction between the viscous flow in the layer very near the wall and the bulk of the flow in the main boundary layer. The displacement $A$ also appears in the expression for the wall skin friction and thus its determination is the main focus of the remainder of this section.

Turning therefore to the inner Goldstein layer, the stream function expansion is once again found by rewriting the oncoming stream function $(2.10 b)$ in terms of the $(X, z)$ coordinate system of our region of interest. This gives

$$
\Psi^{\mathrm{i}}(X, z, T)=h^{-\frac{5}{8}} \Psi_{1}^{\mathrm{i}}(X, z, T)+h^{-1} \Psi_{2}^{\mathrm{i}}(X, z, T)+h^{-\frac{11}{8}} \Psi_{3}^{\mathrm{i}}(X, z, T)+\ldots,
$$

an expansion that is confirmed by rewriting (2.17) as $Y \rightarrow 0$ using $Y=h^{-3 / 8} z$. The condensed flow equation (2.2) in the inner Goldstein layer of the roughness region is

$$
h^{\frac{9}{4}} \frac{\partial^{2} \Psi^{\mathrm{i}}}{\partial T \partial z}+h^{\frac{13}{4}} \frac{\partial \Psi^{\mathrm{i}}}{\partial z} \frac{\partial^{2} \Psi^{\mathrm{i}}}{\partial X \partial z}-h^{\frac{13}{4}} \frac{\partial \Psi^{\mathrm{i}}}{\partial X} \frac{\partial^{2} \Psi^{\mathrm{i}}}{\partial z^{2}}=-h^{\frac{3}{2}} \frac{\partial p}{\partial X}+h^{\frac{21}{8}} \frac{\partial^{3} \Psi^{\mathrm{i}}}{\partial z^{3}}
$$

into which we substitute the expansions (2.19) and (2.14). Each order is then equated to form a governing equation for the terms in the expansion, to which we apply the no-slip boundary condition, matching with the upstream stream function and the outer Goldstein layer. The first two terms are

$$
\begin{aligned}
& \Psi_{1}^{\mathrm{i}}(X, z, T)=\frac{1}{6} \mu z^{3}, \\
& \Psi_{2}^{\mathrm{i}}(X, z, T)=\frac{1}{2} \mu z^{2}[A(X, T)+f(X, T)],
\end{aligned}
$$

with the second order system also yielding the upstream condition on the displacement function $A$,

$$
A(X, T) \rightarrow 2 \mu^{-1} \alpha_{0}|X|^{\frac{1}{2}}-\mu^{-1} A_{0 L}|X|^{-\frac{1}{2}} \ln |X|+\mu^{-1} A_{0}|X|^{-\frac{1}{2}}
$$

as $X \rightarrow-\infty$.

Comparing these with the results of $\mathrm{SD}$, we see that the solution at leading order is the same, with the solution to $\Psi_{2}^{\mathrm{i}}\left(\right.$ their $\left.\bar{\psi}_{1}\right)$ being modified by the addition of $f$. The wall skin friction is then

$$
\tau_{\text {wall }}(X, T)=h^{\frac{3}{4}} \mu(A+f)+\ldots
$$

to leading order and thus we are interested in the streamwise position where

$$
A(X, T)+f(X, T)=0 .
$$

The displacement function $A$ is found through the governing equation for $\Psi_{3}^{\mathrm{i}}$, which is

$$
\frac{\partial^{3} \Psi_{3}^{\mathrm{i}}}{\partial z^{3}}+\mu z \frac{\partial \Psi_{3}^{\mathrm{i}}}{\partial X}-\frac{1}{2} \mu z^{2} \frac{\partial^{2} \Psi_{3}^{\mathrm{i}}}{\partial X \partial z}=\frac{\partial P_{1}}{\partial X}+\frac{1}{2} \mu z^{2}(A+f) \frac{\partial}{\partial X}(A+f)+\mu z \frac{\partial}{\partial T}(A+f),
$$


to be solved subject to the condition of no-slip, upstream matching and the condition as $z \rightarrow \infty$ that

$$
\Psi_{3}^{\mathrm{i}} \rightarrow-\frac{1}{60} \alpha_{0}^{2} z^{5}+\frac{1}{2} A_{2} z^{2}+\frac{1}{2} \mu(A+f)^{2} z+\int_{-\infty}^{X} \frac{\partial}{\partial T}(A+f) \mathrm{d} X
$$

where the function $A_{2}$ is unknown. A similar approach to that carried out by SD, Ruban (1981, 1982), Stewartson (1970), Smith (1982), Stewartson et al. (1982), BK and Braun \& Scheichl (2014) yields the governing equation for the displacement $A$ :

$$
\begin{aligned}
& (A+f) \frac{\partial}{\partial X}(A+f)+\sigma_{1} \int_{-\infty}^{X}(X-s)^{-\frac{1}{4}} \frac{\partial^{2}}{\partial T \partial s}(A+f) \mathrm{d} s+2 \mu^{-2} \alpha_{0}^{2} \\
= & -\sigma_{2} F_{\mathrm{s}} \int_{-\infty}^{X}(X-s)^{-\frac{1}{2}} \frac{\partial^{2} A}{\partial s^{2}} \mathrm{~d} s,
\end{aligned}
$$

subject to the starting condition (2.22). In the above, we have already made use of the pressure-displacement relation (2.18) to obtain the integral term on the right-hand side and $\sigma_{1}, \sigma_{2}$ are constants given by

$$
\sigma_{1}=2^{\frac{5}{4}} \pi^{-1} \mu^{-\frac{3}{4}}\left(-\frac{1}{4}\right) ! \quad \text { and } \quad \sigma_{2}=\pi^{-1} \mu^{-\frac{3}{2}}\left[\left(-\frac{1}{4}\right) !\right]^{2},
$$

both positive.

The affine transformation

$$
\begin{gathered}
A=\left(2 \alpha_{0} \sigma_{2} \mu^{-1} F_{\mathrm{s}}\right)^{\frac{1}{2}} \hat{A}, \quad f=\left(2 \alpha_{0} \sigma_{2} \mu^{-1} F_{\mathrm{s}}\right)^{\frac{1}{2}} \hat{f}, \quad T=\frac{1}{2} \mu \alpha_{0}^{-1} \sigma_{1}\left(\frac{1}{2} \mu \alpha_{0}^{-1} \sigma_{2} F_{\mathrm{s}}\right)^{\frac{1}{4}} \hat{T} \\
X=\frac{1}{2} \mu \alpha_{0}^{-1} \sigma_{2} F_{\mathrm{s}}\left\{\hat{X}-2\left(\mu \sigma_{2} F_{\mathrm{s}}\right)^{-1}\left[\alpha_{0}-A_{0 L} \ln \left(\frac{1}{2} \mu \alpha_{0}^{-1} \sigma_{2} F_{\mathrm{s}}\right)\right]\right\}
\end{gathered}
$$

takes the above equation for $A$ to (removing the overhat)

$$
(A+f) \frac{\partial}{\partial X}(A+f)+\int_{-\infty}^{X}(X-s)^{-\frac{1}{4}} \frac{\partial^{2}}{\partial T \partial s}(A+f) \mathrm{d} s+\frac{1}{2}=-\int_{-\infty}^{X}(X-s)^{-\frac{1}{2}} \frac{\partial^{2} A}{\partial s^{2}} \mathrm{~d} s,
$$

to be solved subject to

$$
A \rightarrow|X|^{\frac{1}{2}}-\frac{1}{2}|X|^{-\frac{1}{2}} \ln |X| \quad \text { as } X \rightarrow-\infty .
$$

This can be compared with similar equations obtained by SD and BK. The former can be recovered from (2.28) by setting $f=0$ and taking the displacement function, in the absence of any time-dependent forcing, to be independent of time, thereby removing the first of the two integrals. As already mentioned, the solution to the resultant equation passes smoothly through the point $A(X)=0$ and encounters a singularity at some finite distance further downstream. In the graphs to come, this 'no-roughness' solution will be represented by a dotted line. The same behaviour is seen in the solution of (2.28) and of interest to us here is, firstly, whether the position of zero skin friction $(A+f=0)$, once the mean over a period of oscillation is taken, is moved upstream or downstream for various roughness positions, widths, heights and oscillation frequencies; and, secondly, whether the singular point can also be moved downstream given an appropriate choice of roughness parameters. Equation (2.9) of BK, their equivalent to our (2.28), shows some fundamental differences, as expected given the different scenarios being considered. We first note the absence of the parameter $\Gamma$-representing, for example, the increment in 
angle of attack of a flow over an airfoil - which is not applicable here, and the change of sign of the hump shape, in part due to a different pressure-displacement relation. We have a nonlinear term in $f$ (which will allow us to write the solution to $A$ as a sum of Fourier modes in $\S 4$ ) and integration of (2.28) with respect to $X$ yields a linear rather than a quadratic term in $X$. Notwithstanding these differences, can our roughness produce similar beneficial effects on the wall skin friction as it produced on the value of $\Gamma_{c}$ for BK? Most especially, are there any similarities in the required choice of values of the parameters listed above?

The roughness height and time scalings of order $h^{-5 / 4}$ and $h^{-11 / 8}$ respectively arise from the requirement that the roughness shape $f$ and the time derivative appear in equation (2.28). Larger heights, of $O\left(h^{-7 / 8}\right.$ ) (i.e. of the same order of magnitude as the inner Goldstein layer), would lead to some form of breakdown in the structure of the flow layers considered above. The expansion of the upper Goldstein layer stream function, (2.15), would contain a term at $O\left(h^{1 / 8}\right)$ given by

$$
\Psi_{\text {new }}^{\mathrm{o}}=\psi_{0 \mathrm{~s}}^{\prime}(Y) f(X, T),
$$

which would modify the matching condition at infinity of $\Psi_{1}^{\mathrm{i}}$ to

$$
\Psi_{1}^{\mathrm{i}} \rightarrow \frac{1}{6} \mu z^{3}-\frac{1}{2} \mu z^{2} f
$$

A solution to the governing equation for $\Psi_{1}^{\mathrm{i}}$ with the above boundary condition could not be found. Smaller roughness heights, of $O\left(h^{-5 / 4}\right)$ are a subset of $(2.28)$ where $f$ is set to zero and the equation of SD recovered. Thus the wall skin friction remains the same as theirs to leading order and the dynamic roughness serves only to slightly modify its position at higher order.

The choice of time scaling comes from a consideration of the condensed flow equation in the inner Goldstein layer and a requirement that the time derivative of $\Psi_{i}^{\mathrm{i}}$ appears in the governing equation for $\Psi_{i+1}^{\mathrm{i}}$. Increasing the oscillation frequency by taking time to be $O\left(h^{-7 / 4}\right)$ would leave the solution to $\Psi_{1}^{\mathrm{i}}$ unchanged, but ensure that the time derivative is included in the governing equation for $\Psi_{2}^{\mathrm{i}}$ :

$$
\frac{\partial^{3} \Psi_{2}^{\mathrm{i}}}{\partial z^{3}}=\frac{\partial^{2} \Psi_{2}^{\mathrm{i}}}{\partial T \partial z}+\frac{1}{2} \mu z^{2} \frac{\partial^{2} \Psi_{2}^{\mathrm{i}}}{\partial X \partial z}-\mu z \frac{\partial \Psi_{2}^{\mathrm{i}}}{\partial X}
$$

with matching condition

$$
\Psi_{2}^{\mathrm{i}} \rightarrow \frac{1}{2} \mu z^{2}(A+f)+\int_{-\infty}^{X} \frac{\partial}{\partial T}(A+f) \mathrm{d} s
$$

as $z \rightarrow \infty$ and the no-slip condition at the surface. In particular, there is no forcing from the pressure perturbation $P_{1}$. Taking $t=h^{-1} T$ would force the time derivative of $\Psi_{i}^{\mathrm{i}}$ to appear in the equation for $\Psi_{i+2}^{\mathrm{i}}$. In particular, since $\Psi_{1}^{\mathrm{i}}$ remains independent of time, there would be no time derivative in the equation for $\Psi_{3}^{\mathrm{i}}$, turning equation (2.28) into

$$
(A+f) \frac{\partial}{\partial X}(A+f)+\frac{1}{2}=-\int_{-\infty}^{X}(X-s)^{-\frac{1}{2}} \frac{\partial^{2} A}{\partial s^{2}} \mathrm{~d} s .
$$

This is precisely the equation for a static roughness element analysed in the next section, $\S 3$. The oscillations of $f$ would then not be able to modify the wall skin friction to leading order. This would recreate, in a different way, the structure of BK, who considered a steady hump with small vibrations to it: this steady hump allowed them to increase the value of $\Gamma_{c}$ (as it will allow us to shift the position of zero skin friction downstream) with adjustments to the wall shear appearing at higher order. 


\section{Static roughness}

One of the conclusions of $\mathrm{BK}$ is that a static roughness, appropriately placed, can increase $\Gamma_{c}$. Here, we consider whether a static roughness, appropriately placed, can shift the position of zero skin friction downstream for flow over a hump. The governing system for the displacement function is (2.30) subject to the upstream condition (2.29). The static roughness shape is given by

$$
f(X)=a\left(X-X_{L}\right)^{4}\left(X-X_{R}\right)^{4}, \quad X \in\left[X_{L}, X_{R}\right],
$$

ensuring that it is sufficiently smooth at the left- and right-hand edges, $X_{L}$ and $X_{R}$ respectively. The constant $a$ is the $O(1)$ scaled height of the roughness and is renormalised by $256\left(X_{L}-X_{R}\right)^{-8}$ to ensure that a choice of $a=1$ gives a maximum height of 1 at the roughness midpoint, $X_{M}$.

To compute the displacement function $A$, an integration by parts was carried out on the integrals to remove the singularity in the integrand at the upper limit of the integration range, with the resultant integral treated by a trapezoidal approximation. Calling $\beta=\partial A / \partial X$ and $\gamma=\partial \beta / \partial X$, a second-order accurate centred difference was used on the derivative in the integral and the resulting discretised equation solved for $\gamma_{n}$, from which one computed $\beta_{n}$ and $A_{n}$, where the subscript $n$ denotes the $n$th mesh point. The nonlinearity was dealt with by using $A_{n-1}$ in the calculation of $\gamma_{n}$ (and hence $A_{n}$ ). Mesh checks were carried out, which showed good agreement for mesh steps between 0.01 and 0.0005 ; a step of 0.001 was used in the production of the graphs that appear in $\S \S 3$ and 4 . The semi-infinite integral was truncated at a finite $X_{1}$ and the leading order term in the upstream matching condition (2.29) used to analytically compute the integral between $-\infty$ and $X_{1}$. The solutions for $X_{1}$ equal to $-4.9,-9.9,-14.9$ and -19.9 were calculated and compared: there was little difference between each one and thus we set $X_{1}=-4.9$ or -9.9 , depending on the position of the roughness element.

The solution for a positive roughness element placed between -5 and -1 , with height 1 (dashed line) is shown in figure $2 a$, compared with the solution in the absence of a roughness (dotted line). Both the displacement function (dot-dash line) and wall skin friction (solid line) are given. We first note that in this case the position of zero skin friction has advanced slightly compared to the no-roughness case and that the singular position, linked to the appearance of a still shorter length scale over which the full nonlinear breakaway of the shear layer occurs, has also moved forwards. Focusing on the solution over the roughness element, which has been placed well upstream of the original zero skin friction point, we note that the displacement first decreases over the front face of the roughness, before recovering slightly over the rear. Referring back to the pressure-displacement relation (2.18), which equates displacement with pressure (ignoring the positive constant $F_{\mathrm{s}}$ ), this makes physical sense: where the roughness is increasing in height, we encounter a favourable pressure gradient, and so pressure (and, here, displacement) decreases and the flow speeds up, resulting in an increase in the skin friction. The reverse occurs where the roughness decreases in height: the pressure gradient is adverse, the displacement increases, the flow is retarded and the skin friction decreases. The same behaviour, but in the opposite order, occurs for negative roughness heights, as shown in figure $2 b$.

The equation for the skin friction (2.23) suggests the possibility of small separation bubbles existing within the flow when the roughness is negative $(f<0)$, for sufficiently large roughness depth. This is shown in figure $3 a$, with the skin friction going negative but then recovering. A small increase in the depth, however, causes a bursting of the bubble and the sudden dramatic advancement of the singular point (figure $3 b$ ). Interestingly, 


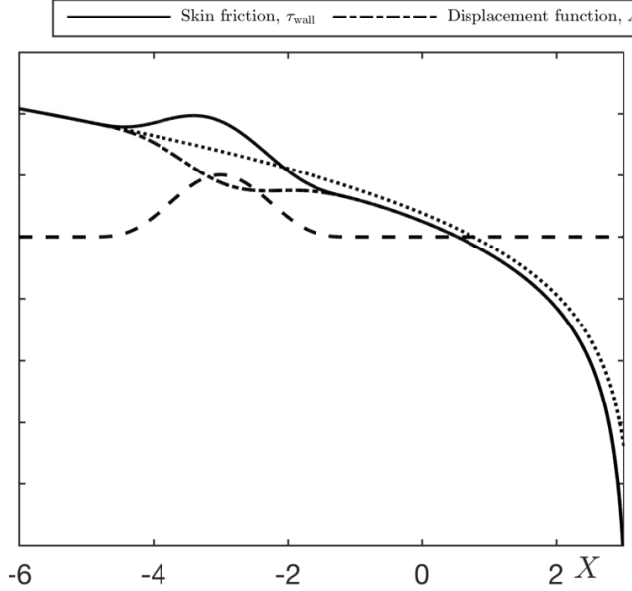

(a)

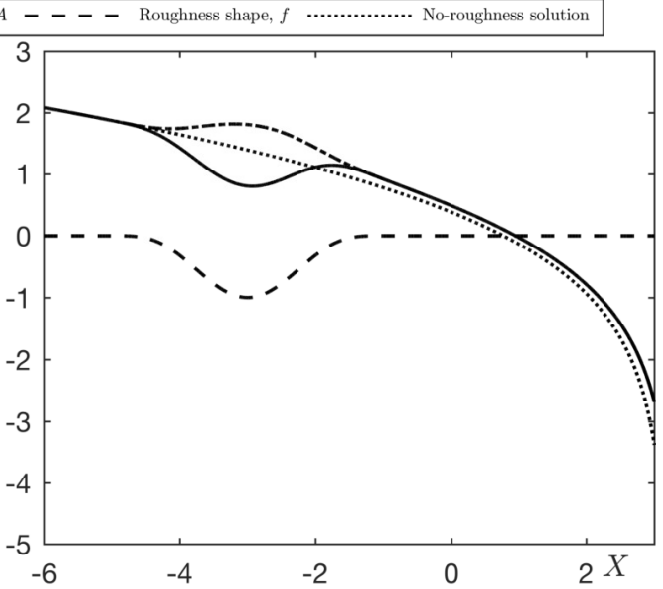

(b)

Figure 2. Solution for a static roughness, either positive $(a)$ or negative $(b)$, placed between $X_{L}=-5$ and $X_{R}=-1$ and of height 1 (dashed line), with the skin friction shown by the solid line and the displacement by the dot-dashed line. These are compared with the solution to the no-roughness case (dotted line), which is the same as that of SD, where the skin friction and displacement are the same.

this very large upstream movement of the singular point has not been seen for positive roughness elements. Physically speaking, at lower roughness depths, local separation over the front, backwards-facing half of the roughness occurs, and the flow reattaches itself onto the rear, forwards-facing half, with a small separation bubble existing in the region of negative skin friction. As the depth increases, however, the local separation position moves upstream and eventually, at sufficiently large depth, the separated streamline is no longer able to reattach and full breakaway separation, indicated by the singularity, instead occurs. Narrower roughness elements admit larger depths.

The addition of the roughness function $f$ to the displacement $A$ to obtain the skin friction indicates that in order to shift the position of vanishing skin friction downstream, a positive roughness must lie over the original zero skin friction position, while a negative roughness should not. Figures 4 and 5 plot the shift in the local separation point for varying roughness positions (represented by their midpoint) and widths (the height throughout was set equal to one), compared to where no roughness is present. A positive number (represented by a shade of red) corresponds to a downstream movement; while a negative number (shade of blue) is an upstream movement. The first image clearly shows the desirability of placing the roughness such that its midpoint occurs just downstream of the original point of zero skin friction, with significant downstream shifts observed, both in the zero skin friction position and the singular point, especially for increasing roughness width. A clear demarcation between a downstream and upstream shift in the position of zero skin friction is seen in figure 5 for a negative roughness. This is due to the small separation bubble referred to previously 'bursting', i.e. the skin friction initially goes negative, reaches a local minimum, recovers, but not sufficiently to become positive again.

This dependence on roughness parameters - positive roughness near the point of zero skin friction with a preference for wider roughness elements; negative roughness upstream of the point of zero skin friction - agrees with that found by BK for the increase in $\Gamma_{c}$, although it is not possible to separate the individual impact of position and width from 


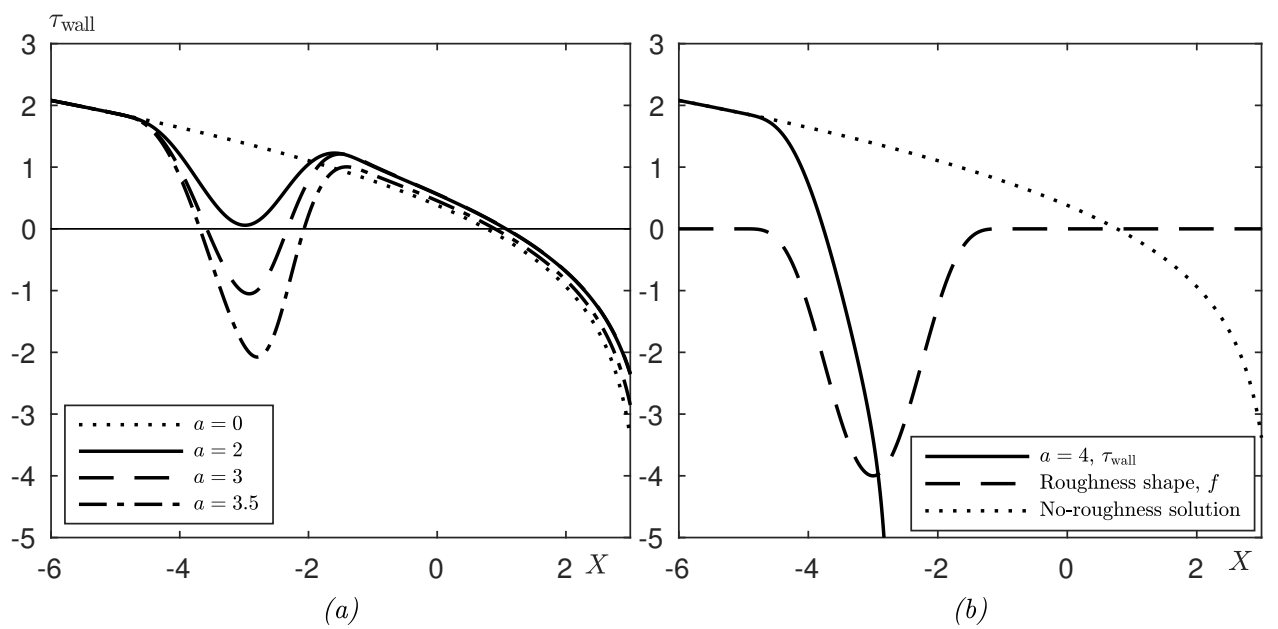

FiguRE 3. Effect of the gradual increase in depth on the solution for where a negative static roughness centred at $X_{M}=-3$ of width 4 is present. In $(a)$, the skin friction is plotted for depths of $a=2$ (solid line), $a=3$ (dashed) and $a=3.5$ (dot-dashed); in (b), the depth is $a=4$ and plotted are the displacement function (solid line) and roughness shape (dashed line). In both, the dotted line is the no-roughness solution.

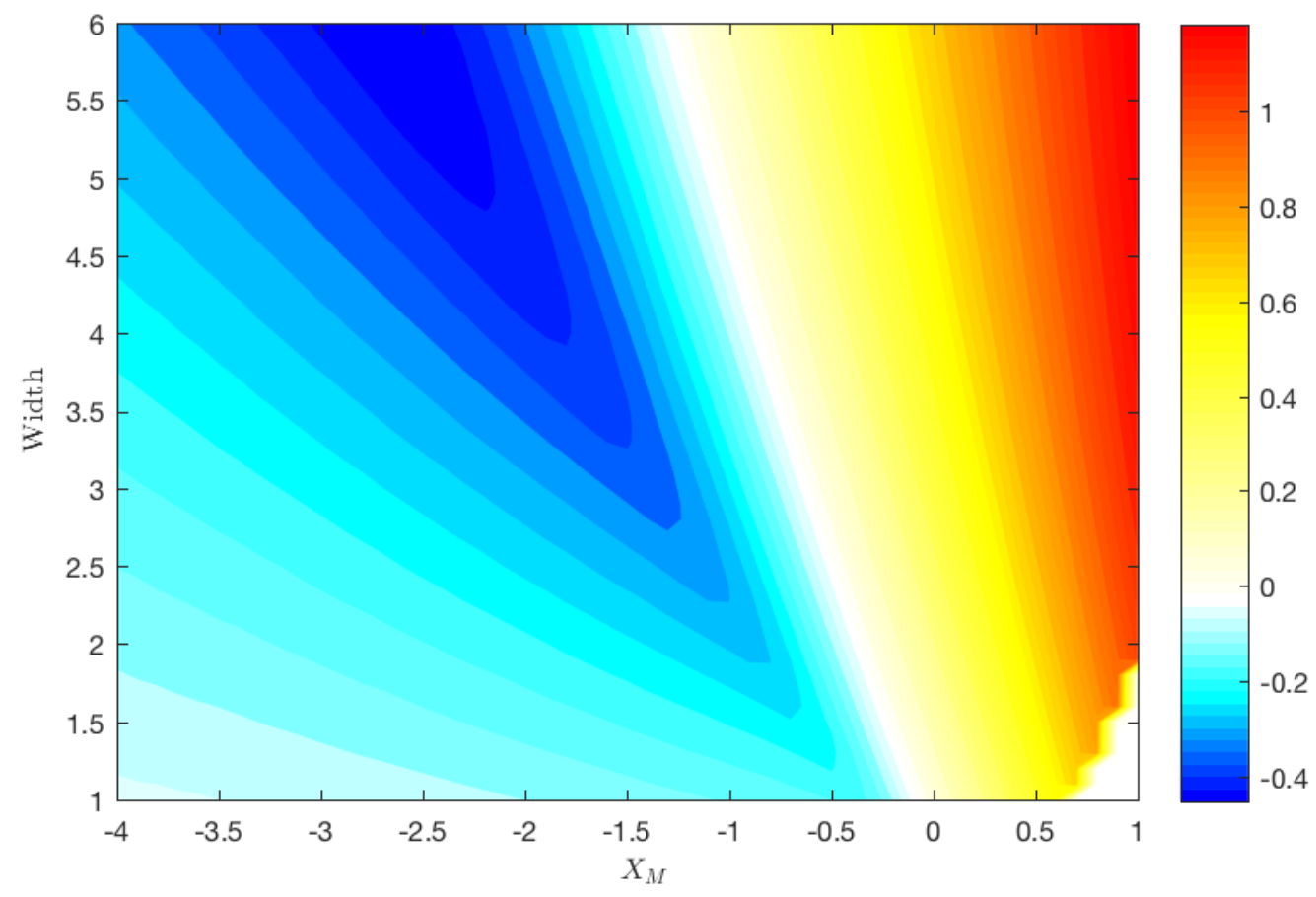

FIGURE 4. Influence of position and width of a positive roughness element on the position of zero skin friction, compared to when no roughness is present. A downstream shift is represented by positive numbers (red), while an upstream shift are negative numbers (blue). The roughness height was taken to be 1. 


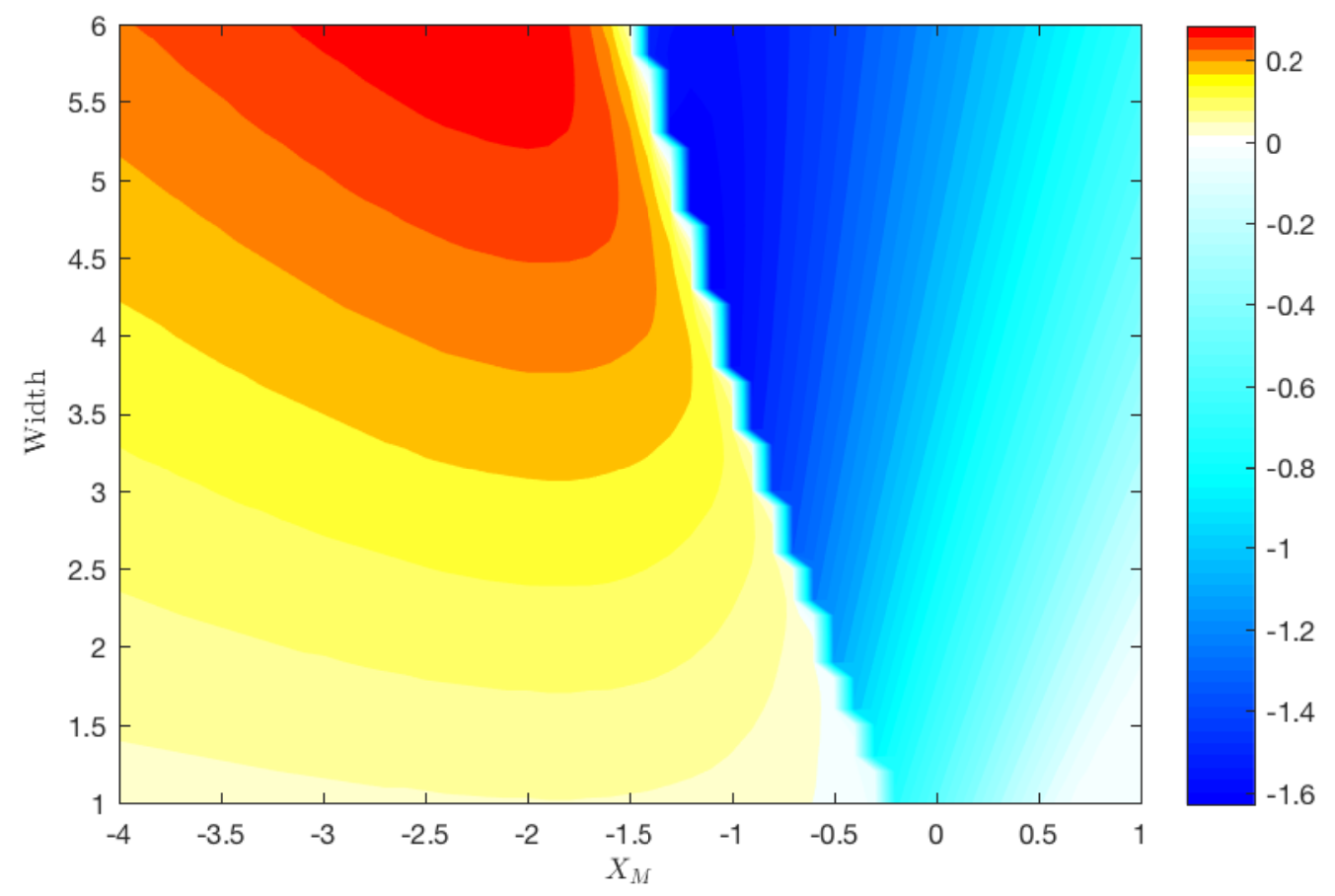

FIGURE 5. As in figure 4, but for a negative roughness element.

the results reported in their paper. Figure 6 indicates why a wider bump is beneficial. With the slope of the roughness diminishing, the gradient of the pressure (or, equivalently, displacement) over the front face changes only slightly compared to the no-roughness case; once the roughness peak is passed, one would expect the pressure gradient to become adverse, but the encroaching negative singularity prevents this occurring, allowing it only to become less favourable. An inflection point in $A$ therefore occurs, resulting in the singularity being shifted downstream, possibly delaying the occurrence of full, nonlinear, breakaway separation. Here we encounter the counter-intuitive nature of the present pressure-displacement relation, to which we will return in our closing comments in $\S 6$.

Increasing the height of a positive roughness element is beneficial to the downstream movement of the position of zero skin friction, provided that the position and width of the roughness are such that it does move to the right (see figure 7 ). For the roughness midpoints plotted, a saturation point is reached as the singular point cannot move downstream indefinitely and will eventually force the skin friction to become negative. Moving the midpoint further downstream, as presented in $\S 5$, will, however, result in a significant downstream shift of the singular point.

\section{Dynamic roughness}

Equation (2.28) for the displacement function $A$ can be rewritten as a system for $A+f$, which is effectively the wall skin friction (c.f. (2.23)), giving

$$
\tau \frac{\partial \tau}{\partial X}+\int_{-\infty}^{X}(X-s)^{-\frac{1}{4}} \frac{\partial^{2} \tau}{\partial T \partial s} \mathrm{~d} s+\int_{-\infty}^{X}(X-s)^{-\frac{1}{2}} \frac{\partial^{2} \tau}{\partial s^{2}} \mathrm{~d} s=\int_{-\infty}^{X}(X-s)^{-\frac{1}{2}} \frac{\partial^{2} f}{\partial s^{2}} \mathrm{~d} s-\frac{1}{2}
$$




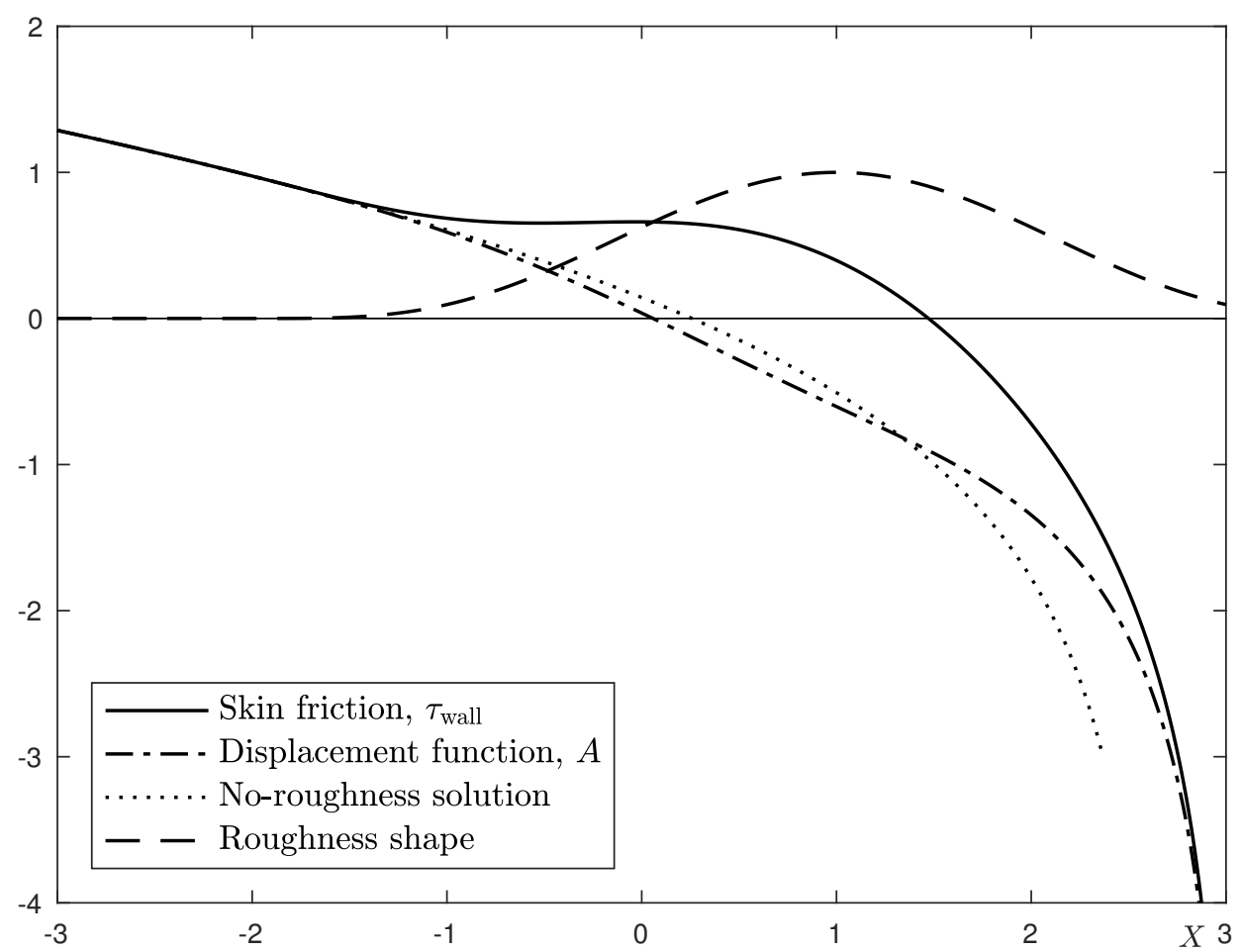

FIGURE 6. A positive static roughness of height 1 and width 6 , centred at $X_{M}=1$ (dashed line), with the associated skin friction (solid line) and displacement function (dot-dashed). The no-roughness solution is given by the dotted line for comparison.

with the same upstream behaviour as (2.29) since the roughness element is absent there. For ease of notation, we drop the subscript 'wall' when referring to the scaled skin friction throughout this section, on the understanding that by $\tau$ we mean $\partial^{2} \Psi^{\mathrm{i}} / \partial y^{2}$ evaluated at $y=0(z=0)$.

The time dependence in the work of BK appeared at higher order in their expansion of the wall shear, since their dynamic roughness element took the form of low amplitude vibrations around a steady hump shape. This correction to the leading order wall shear was obtained in part as the solution to the forced Fisher equation - forced by the form of the obstacle vibrations and the leading order solution - and their main interest in it was as an analysis of the bursting of the small separation bubble as a result of the finite time blow-up of the governing equation. Indeed, it is well known that equation (4.1) is ill-posed and that a numerical solution through time marching will lead to a singularity at some finite time $T_{0}$, when the magnitude of the displacement function $A$ becomes arbitrarily large at some streamwise position (Smith 1982; Braun \& Kluwick 2004).

Given this, we tackle (4.1) by writing the skin friction $\tau$ as the sum of Fourier modes, with the solution to each mode being forced by the fixed frequency oscillations of the dynamic roughness function and the nonlinearity present. Our interest remains in keeping the time dependence at leading order and thereby seeing whether a roughness whose oscillation amplitude is the same as its maximal extension can shift the local and breakaway separation points downstream and, if so, whether it is more or less effective than a static roughness. This fundamental difference from the work of BK perhaps makes comparison between our results and theirs difficult. In addition, the 


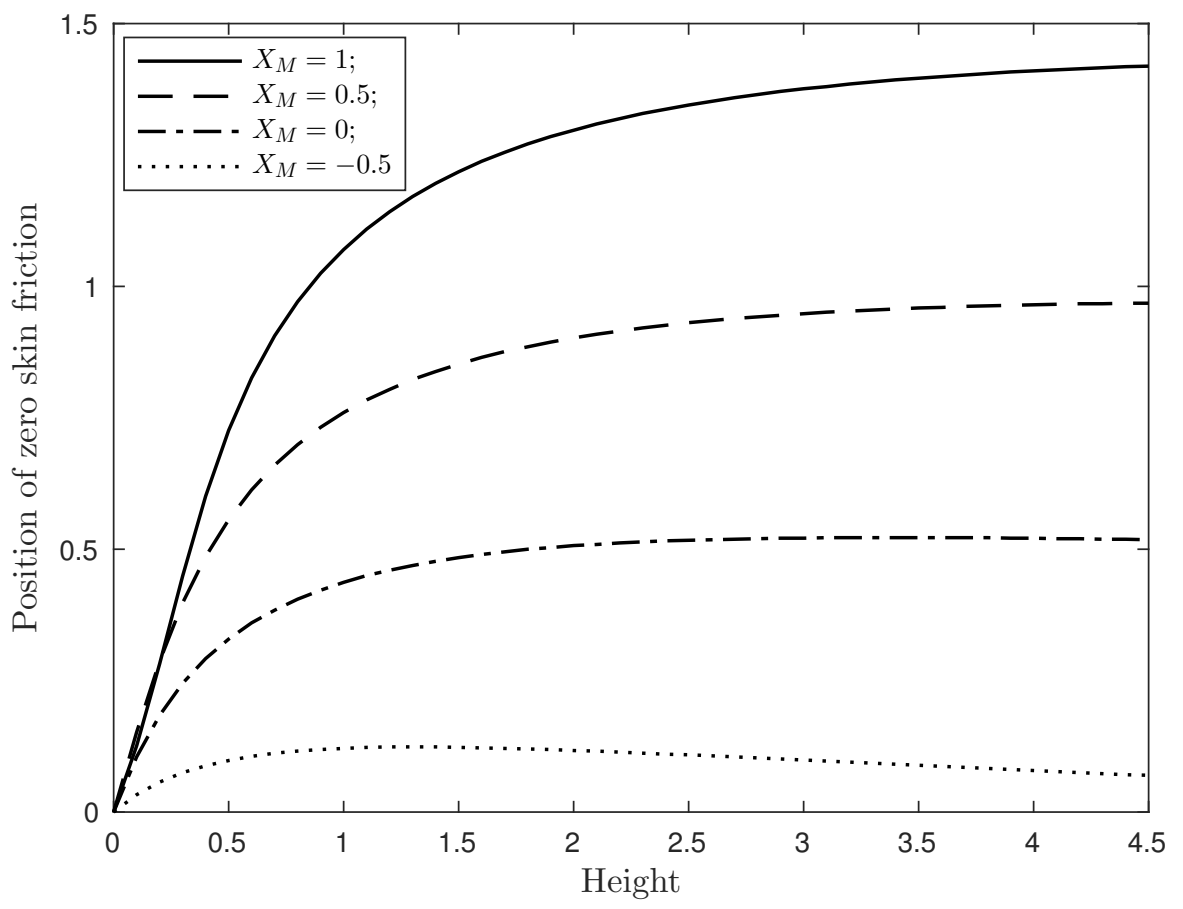

FiguRE 7. Impact of positive roughness height on the local separation position, for various positions of the roughness midpoint (the width was set equal to 4): $X_{M}=1$ (solid line), $X_{M}=0.5$ (dashed), $X_{M}=0$ (dot-dashed) and $X_{M}=-0.5$ (dotted).

present model explores the alternative indicated by the experiments described in the introduction, namely of fixed-frequency forced behaviour rather than time-marching, thus offering a different route to transition further downstream.

We write, therefore, the dynamic roughness function as

$$
f(X, T)=g(X)\left(\mathrm{e}^{2 \mathrm{i} \omega T}+\mathrm{e}^{-2 \mathrm{i} \omega T}+2\right)
$$

in order to keep the roughness either positive or negative, depending on the sign of $g$, throughout a cycle of oscillation (roughness elements with no restriction on sign were also analysed). The function $g$ is the basic shape of the roughness, defined precisely later. The skin friction is then written as

$$
\tau(X, T)=\sum_{k=-M}^{M} \tau_{k}(X) \mathrm{e}^{k \mathrm{i} \omega T},
$$

where the sum of Fourier modes has been terminated at some finite $M$, with all higher modes being negligibly small. Our main interest lies in the zeroth Fourier mode, $\tau_{0}$, as this is time independent and hence the only nonzero term remaining once the mean in time has been taken over a period of oscillation (denoted by angled brackets throughout): it gives, therefore, the average position of the point of zero skin friction. Hence $M$ will be chosen by the numerical scheme as the value at which

$$
\max _{1 \leqslant n \leqslant N_{\mathrm{s}}}\left\{\tau_{0}^{(M+2)}\left(X_{n}\right)-\tau_{0}^{(M)}\left(X_{n}\right)\right\}<\text { tolerance, }
$$

where the superscript denotes the number of Fourier modes used and $N_{\mathrm{s}}$ is the number of streamwise mesh points up to the position where $\tau_{0}$ is first less than some value $\tau_{\mathrm{s}}$, 
with $\tau_{\mathrm{s}}$ representing the eventual singular behaviour of the skin friction. In this section, $\tau_{\mathrm{s}}=-3$. Note that all odd Fourier modes will be zero.

The governing equation for each mode $m$ then becomes

$$
\begin{aligned}
& \sum_{k=-M}^{M} \tau_{k} \frac{\partial \tau_{m-k}}{\partial X}+m i \omega \int_{-\infty}^{X}(X-s)^{-\frac{1}{4}} \frac{\partial \tau_{m}}{\partial s} \mathrm{~d} s+\int_{-\infty}^{X}(X-s)^{-\frac{1}{2}} \frac{\partial^{2} \tau_{m}}{\partial s^{2}} \mathrm{~d} s \\
= & \delta_{0 m}\left(-\frac{1}{2}+2 \int_{-\infty}^{X}(X-s)^{-\frac{1}{2}} \frac{\partial^{2} g}{\partial s^{2}} \mathrm{~d} s\right)+\delta_{2 m} \int_{-\infty}^{X}(X-s)^{-\frac{1}{2}} \frac{\partial^{2} g}{\partial s^{2}} \mathrm{~d} s,
\end{aligned}
$$

subject to the upstream condition

$$
\tau_{m} \rightarrow\left\{\begin{array}{ll}
|X|^{\frac{1}{2}}-\frac{1}{2}|X|^{-\frac{1}{2}} \ln |X|, & \text { if } m=0 ; \\
0, & \text { if } m \neq 0
\end{array} \quad \text { as } X \rightarrow-\infty .\right.
$$

Here, $\delta_{i j}$ equals 1 if $i=j$ and 0 otherwise. The system is again treated as spatially parabolic. To solve at the mesh point $X_{n}$, an integration by parts is carried out on the integrals, and the terms involving $\tau_{m}$ are removed from the summation in the left-hand side, with the remaining terms moved over to the right-hand side and evaluated at the $(n-1)$ th mesh point, with a backwards difference used on the derivatives. The method is then similar to that described for the static roughness, since equation (4.5) is turned into an equation for $\partial \tau_{m}^{2} / \partial X^{2}$. A centred difference is used on the derivative in the second integral and all Fourier modes have to be determined at each mesh point before proceeding on to the next one. The semi-infinite integral is dealt with in the same way as described in $\S 3$ and, once again, checks for mesh sizes of $\Delta=0.01,0.001$ and 0.0005 showed good agreement.

The shape function $g$ again has to be chosen such that it is sufficiently smooth at the end points: in order to guarantee this, the function

$$
g(X)=a\left(X-X_{L}\right)^{6}\left(X-X_{R}\right)^{6}, \quad X \in\left[X_{L}, X_{R}\right]
$$

was used. The amplitude $a$ was normalised by $\left(X_{M}-X_{L}\right)^{-6}\left(X_{M}-X_{R}\right)^{-6}$. The solution for small $a$ can be compared with a linearised theory for the governing equation (2.28): a sample comparison is shown in figure 8 and agrees well until the singular position is approached, when the linearised expansion in powers of the small amplitude $a$ is no longer valid.

The solution for the zeroth Fourier mode, which equates to the time-averaged solution, is given in figure 9 for a dynamic roughness with midpoint at $X_{M}=-3$, width 4 , amplitude 1 and oscillation frequency 1 . We concentrate in the remainder of this article on positive roughness only. The behaviour over the dynamic roughness is qualitatively similar to that observed for a static roughness and again agrees with what one would expect physically from the form of the pressure-displacement relation (2.18). Compared to the no-roughness case, the displacement function decreases over the front face of the roughness (skin friction increases) and increases slightly over the rear (skin friction decreases). The most obvious difference between the static and dynamic case is the marked advance of the singular position, which always appears towards the trailing edge of the roughness and occurs rather suddenly. As one increases the frequency $\omega$, this effect becomes more pronounced.

The equation for the time-averaged skin friction,

$$
\langle\tau\rangle=\langle A(X, T)\rangle+2 g(X)=A_{0}(X)+2 g(X),
$$

once more suggests that the dynamic roughness should be placed over the original position 


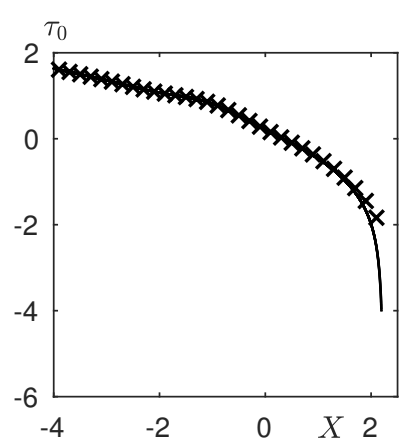

(a)

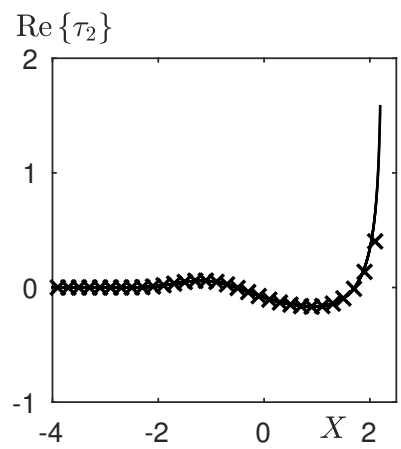

(b)

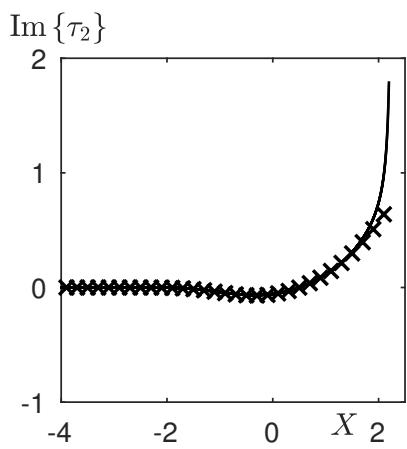

(c)

Figure 8. Comparison between the numerical solution to equation (4.1) for small roughness amplitude, $a=0.1$ (crosses), and the linearised approach (solid line). In both, the roughness was placed between -3 and 1 and had frequency $\omega=1$. The solutions to the steady mode and the real and imaginary parts of the second mode are shown in $(a),(b)$ and $(c)$ respectively.

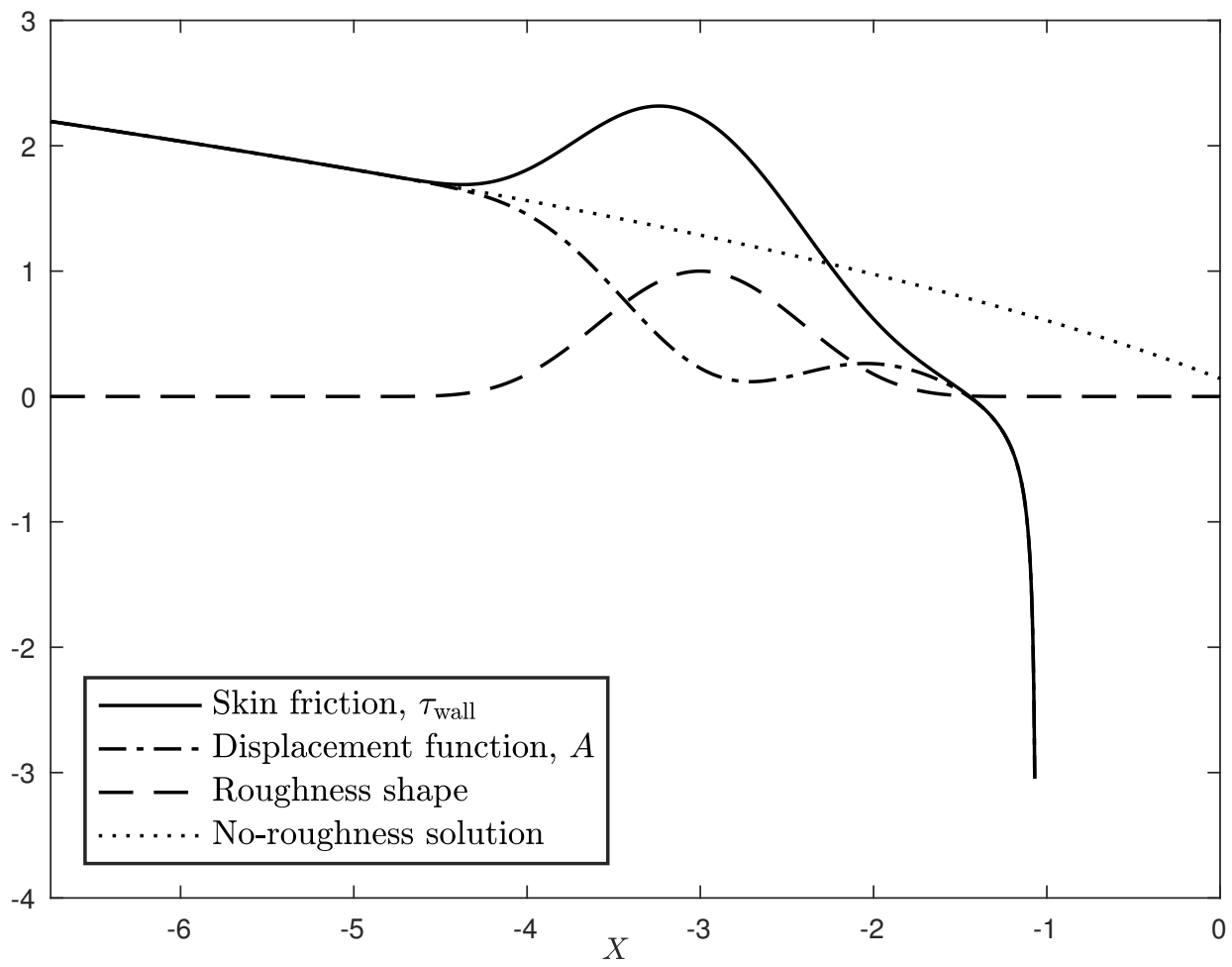

Figure 9. Solution for a positive dynamic roughness, placed between -5 and -1 (dashed line), in terms of the skin friction (solid) and displacement function (dot-dashed), compared with the solution for no roughness present (dotted), when the skin friction and displacement are the same.

of vanishing skin friction in order to move it downstream. This is quite clearly shown in figure 10. The most favourable effect is felt with the front half of the dynamic roughness over the original zero skin friction point, although there must be an optimal position for the roughness (dependent also on its width, height and frequency), since once positioned 


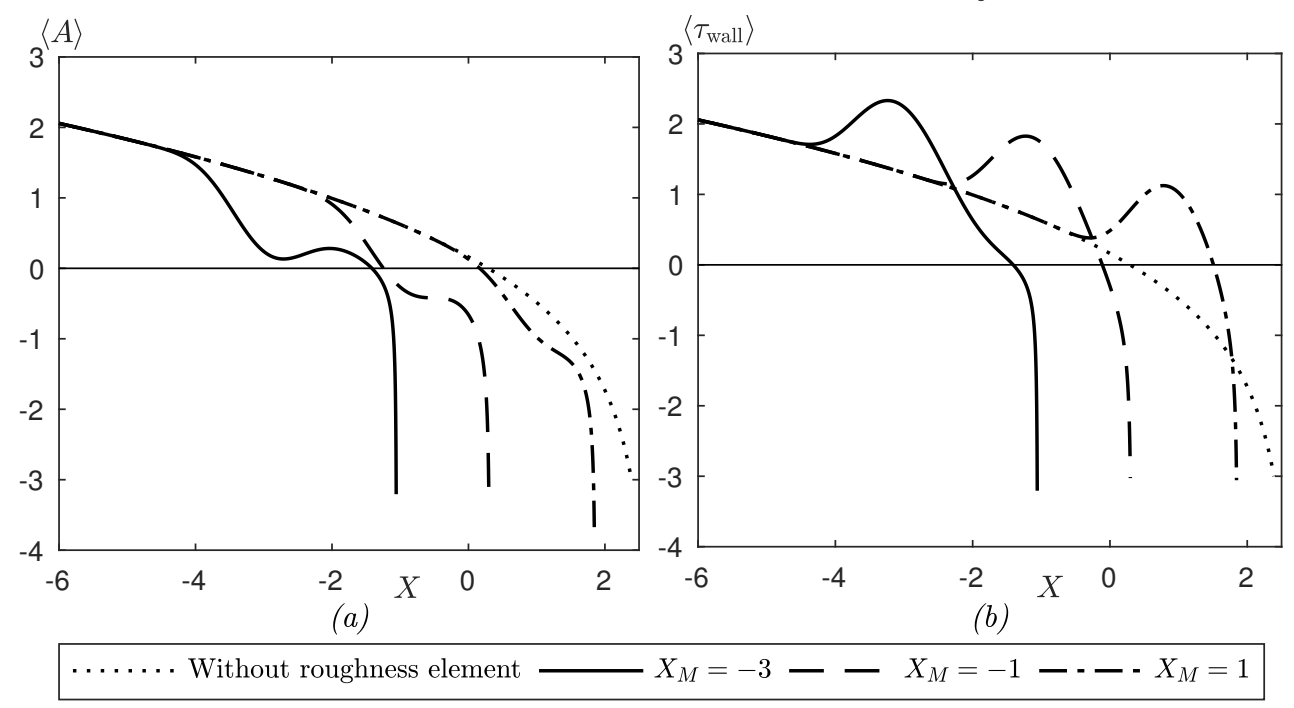

FiguRE 10. Impact of the dynamic roughness position on the displacement function $(a)$ and skin friction $(b)$, with $\omega=1$ and the roughness width fixed at 4 . The roughness position is represented by its midpoint $X_{M}=-3$ (solid line), -1 (dashed) and 1 (dot-dashed). The no-roughness solution is given by the dotted line.
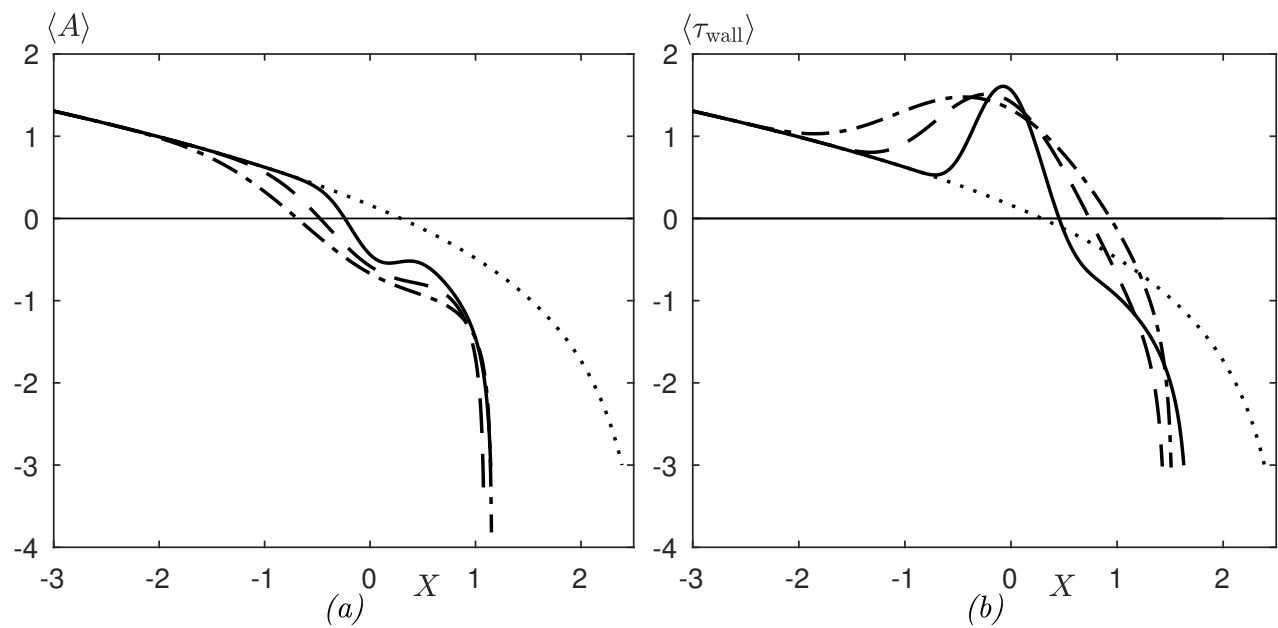

Without roughness element

Width $=2-$ Width $=4--$ - Width $=6$

Figure 11. Impact of the dynamic roughness width on the displacement function $(a)$ and skin friction $(b)$, with $\omega=1$ and the roughness midpoint fixed at $X_{M}=0$. The widths 2 (solid), 4 (dashed) and 6 (dot-dashed) were looked at, with the no-roughness solution given by the dotted line.

completely downstream, the apparent parabolic nature of the governing equation means that the roughness will have no impact on the local separation point. As for a static roughness, an increase in width also increases the delay in the position of zero skin friction (figure 11).

The effect of height and frequency of the roughness element seems heavily interlinked: at low amplitudes, there is little difference in the position of zero skin friction at different frequencies, with changes only becoming clear as $a$ is increased. Similarly, for low $\omega$, 


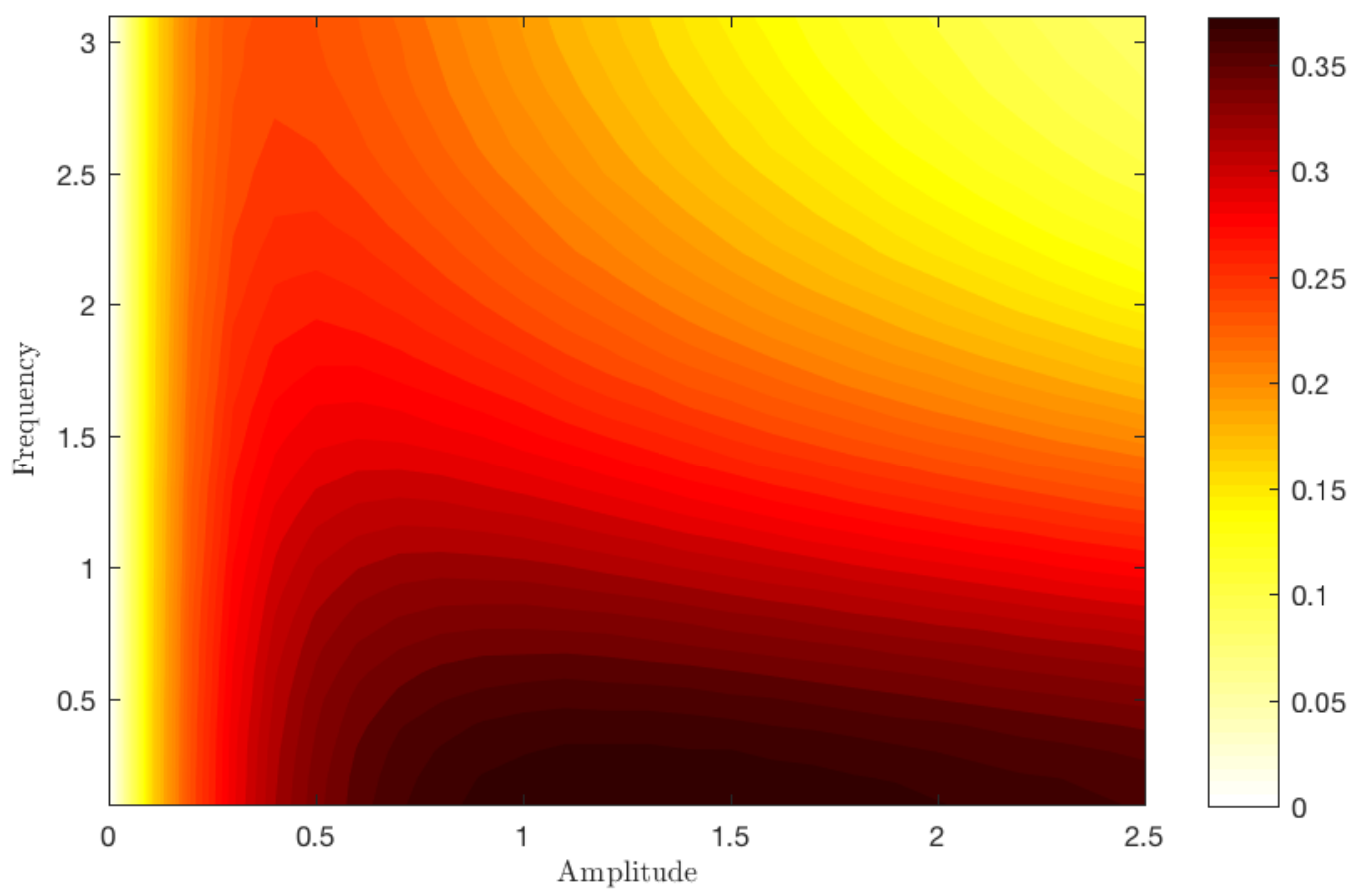

Figure 12. Impact of the frequency $(\omega)$ and amplitude $(a)$ of a single, positive dynamic roughness placed between -2 and 2 on the position of vanishing skin friction, compared to when the roughness is not present. The amplitude was varied from $a=0$ to $a=2.5$ and the frequency from $\omega=0.1$ to $\omega=3.1$, both in steps of 0.1 . The positive numbers seen throughout indicate a downstream shift of the local separation point.

there can be little change in the zero skin friction position for a wide range of amplitudes. Overall, figure 12 (produced for $X_{M}=0$ and width equal to 4 ) shows that above a certain amplitude, there is an inverse relationship between $\omega$ and $a$ : to maintain a given shift in the position of vanishing skin friction, an increase in amplitude has to be compensated by a decrease in frequency and an increase in frequency has to be matched by a decrease in amplitude. We note also that a downstream shift is always seen and that high oscillation frequencies have less of a beneficial impact.

This of course prompts the question as to whether a static or dynamic roughness is better with regard to shifting the zero skin friction position in the mean. The answer is dependent on the choice of roughness parameters: for the selection $X_{L}=-1, X_{R}=3$, $a=1, \omega=1$ (figure 13), the dynamic roughness is superior, although we note that the singular point is advanced. Comparing the graphs of the time-averaged displacement function, the dynamic roughness introduces a greater favourable pressure gradient into the flow over its front face; the increased shift in the point where $\tau_{\text {wall }}=0$ is due to the addition of two times the roughness shape function to $A$, rather than just one.

\section{Breakaway separation}

The downstream singularity that follows the position of zero skin friction throughout is linked to the occurrence of the full nonlinear separation of the boundary layer from the hump surface. In SD, this singularity is removed and the flow development described by considering a region of order $h^{-2}$ length about the singular point (region D of figure 1). 


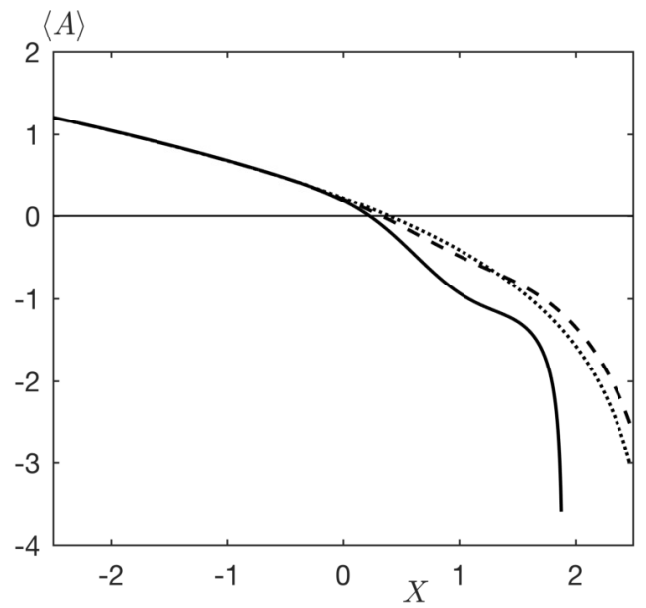

(a)

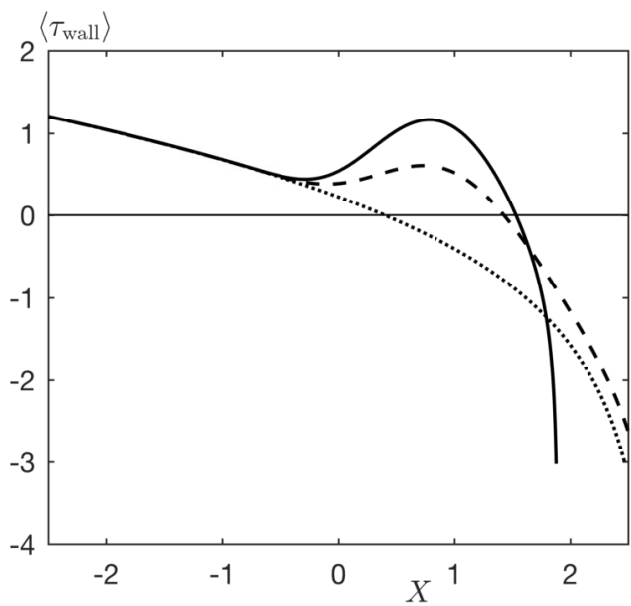

(b)

Figure 13. Comparison in the displacement function $(a)$ and skin friction $(b)$ for a dynamic (solid line) and static (dashed line) roughness, placed between -1 and 3 and of height 1 . For the dynamic roughness, the frequency of oscillation was $\omega=1$. The solution in the absence of a roughness element is given by the dotted line.

Here, we are interested in the possibility of static or dynamic roughness elements moving the singularity downstream, thereby delaying the separation of the boundary layer from the hump surface. Physically, we aim to introduce a large favourable pressure gradient to the flow, expecting that this would serve to prolong the streamwise extent for which the flow is attached. Mathematically speaking, we equate this to a downstream shift of the singular point, $X=X_{\mathrm{s}}$, which can be defined numerically as occurring at the mesh point $n$ where $\left|A_{n+1}-A_{n}\right|$ is greater than some moderately large number, taken to be 5 .

To this end, we place a roughness element, whose shape is again given by the polynomial form of equation (4.7), with midpoint after the position of the no-roughness singularity, but with left endpoint upstream of it. In the graphs that follow, $X_{M}=6$ and $X_{L}=0$. The maximum roughness height is chosen to be larger than those considered previously $(a=5,10,15)$, but we recall that the roughness is still small (of $\left.O\left(h^{-5 / 4}\right)\right)$ compared to the amplitude of the hump $(O(h))$ and the inner Goldstein layer in the roughness region $\left(O\left(h^{-7 / 8}\right)\right)$. We hope then that the considerable favourable pressure gradient introduced over the front face of the roughness delays the breakaway separation of the boundary layer.

We see that for a static roughness element this is indeed the case (figure 14), with the singular position delayed for all roughness heights shown. We note, however, that for $a=5$ (solid line), the singularity still occurs on the front side of the roughness element, with it being shifted to the rear side of the roughness element $\left(X_{\mathrm{S}}>6\right)$ only once the roughness height (and hence gradient) is sufficiently large. Comparing the graphs for $a=10$ and $a=15$, and recalling the pressure-displacement relation $P_{1}=F_{\mathrm{s}} A$ (equation (2.18)), we see firstly that the larger height gives rise to a more favourable pressure gradient over the front half of the roughness, as would be expected; and, secondly, that there is a slight pressure recovery over the rear face of the roughness $(a=10)$ before the singularity is encountered, again as would be expected. The eventual singular point is similar for both $a=10$ and $a=15$ and we suggest that this may be due to the adverse pressure gradient encountered on the lee side of the roughness being greater for 


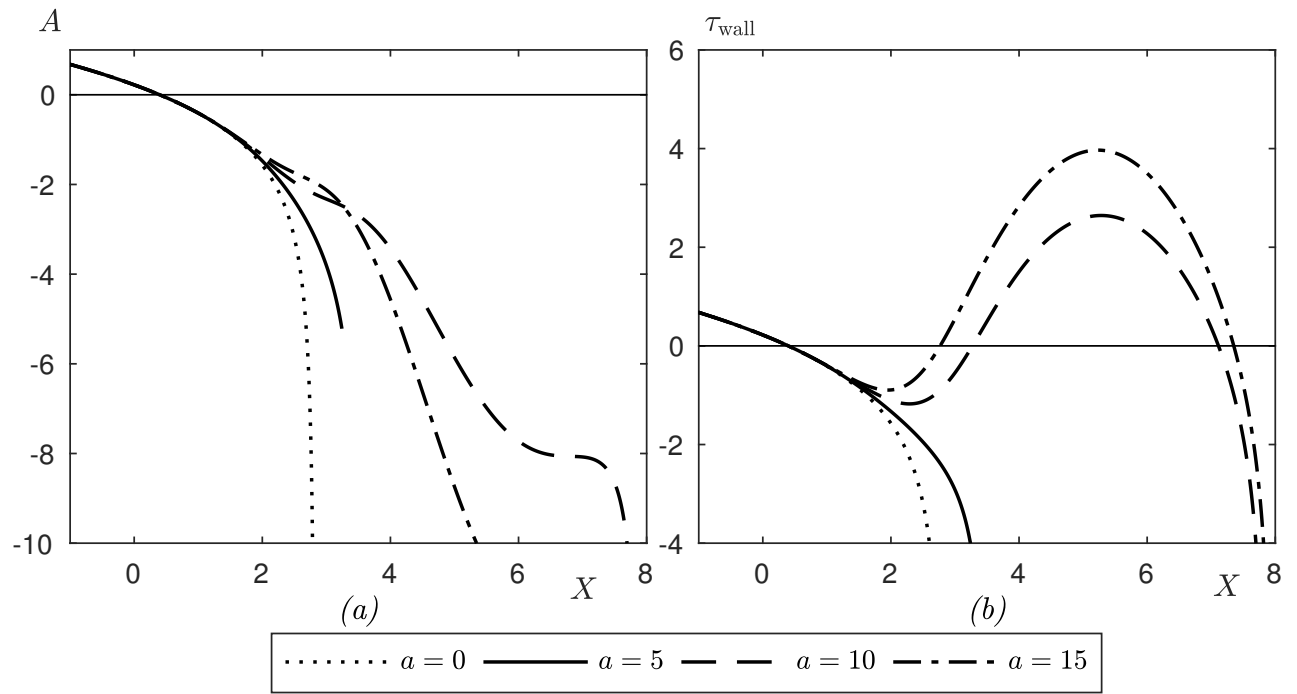

FiguRE 14. Impact of the roughness height on the displacement function $(a)$ and skin friction (b) for a static roughness with left endpoint at $X_{L}=0$ and maximum height at $X_{M}=6$. The roughness shape was as given in equation (4.7). The dotted line represents the no-roughness solution.

increasing height: the effect of an increased acceleration of the flow over the first half of the roughness element being attenuated somewhat by the larger adverse pressure gradient encountered over the second half. In fact, at greater roughness amplitudes, the singular point on the lee side of the roughness element starts moving upstream. For the roughness configuration shown here, we note also the existence of a small region of negative skin friction for the amplitudes $a=10$ and $a=15$.

For dynamic roughness elements, the same numerical method as that described in $\S 4$ was used to obtain the solution for the skin friction, although many more Fourier modes $(M)$ needed to be incorporated into the sum (4.3) to ensure the correct position of the singularity. In condition $(4.4), \tau_{\mathrm{s}}=-5$, with the skin friction decreasing rapidly after this; and the tolerance was typically less than $O\left(10^{-10}\right)$. The increased value of $M$ required meant that the step size was increased to $\Delta=0.005$ to speed up the computation.

Figures 15-17 show that dynamic roughness elements placed with midpoint downstream of the no-roughness singular point but with left-end point upstream of it are also able to shift the position of the singularity to the right and thus delay the breakaway of the boundary layer from the surface, although not to the same extent as static elements. In particular, the singular point remains on the front face of the roughness element at all heights and oscillation frequencies studied. As per static elements, a greater downstream movement is seen for increasing height; and higher frequencies also have a more beneficial effect. This is to be compared with the conclusions of $\S 4$, where an increase in frequency resulted in a diminished downstream shift of the local separation point. For these larger dynamic roughness elements, centred downstream of the no-roughness singular point, we obtain qualitative agreement with the findings of Huebsch et al. (2012), who concluded that an increase in frequency allowed one to decrease the roughness height while still maintaining effective flow control. We note that as $\omega \rightarrow 0$, we do not approach the static solution and instead the singularity moves towards its original, no-roughness position. This is due to the influence of the higher Fourier modes, not present in the static solution 


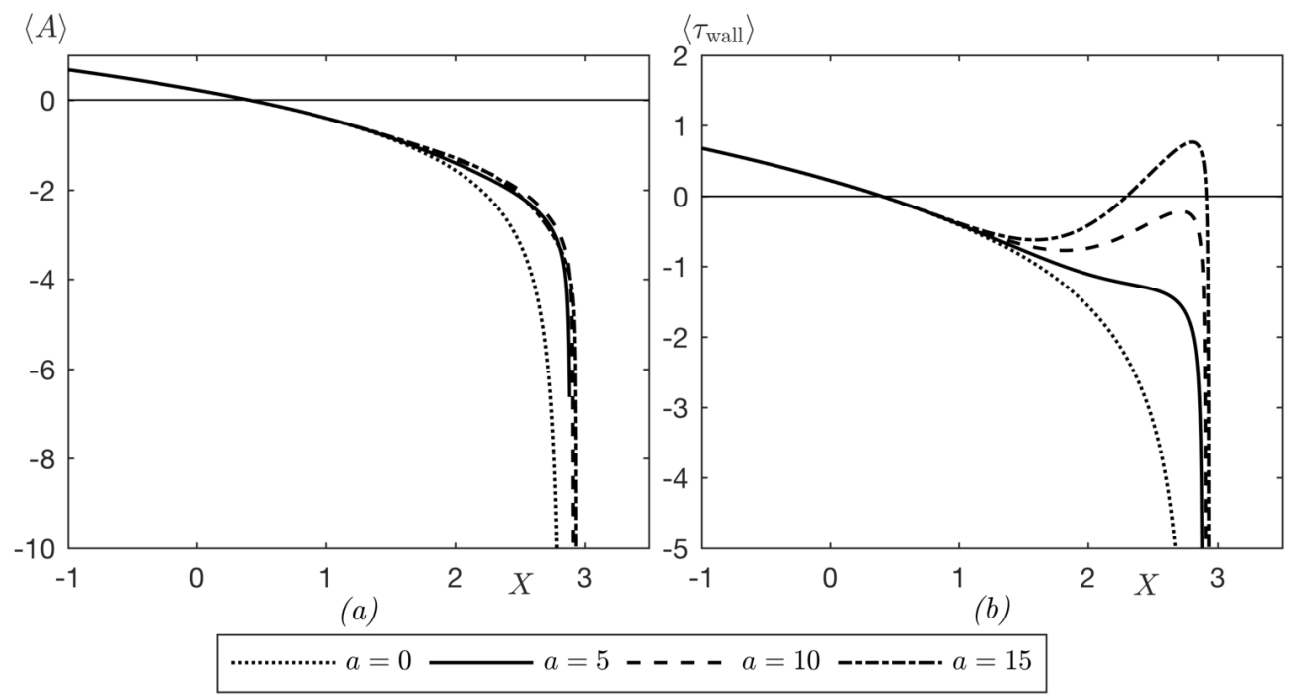

Figure 15. As in figure 14, but for a dynamic roughness oscillated with frequency $\omega=0.5$.

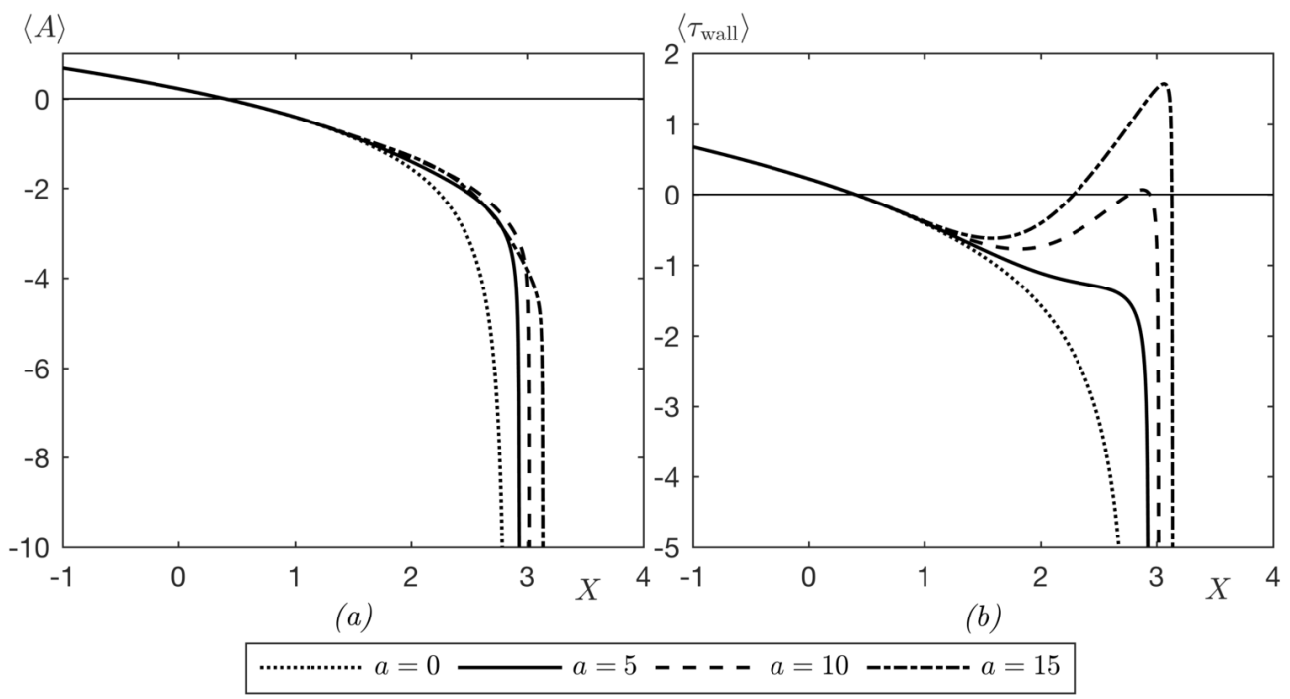

FiguRE 16. As in figure 14, but for a dynamic roughness oscillated with frequency $\omega=1$.

and we believe that, physically, this is due to the roughness element dropping flush with the hump surface before returning to its maximum extension over one cycle of oscillation.

\section{Conclusions}

Motivated by the use of dynamic roughness elements as a potential means of laminar flow control, we have looked at their impact on the position of vanishing skin friction and breakaway separation found by Smith \& Daniels (1981) for flow over a smooth hump embedded within the classical Prandtl boundary layer. In such a scenario, the Goldstein singularity that accompanied the local separation point could be removed and the development of the entire flow as it went over the hump described by a sequence of matched asymptotic expansions in various regions of the flow. The presence of a roughness 


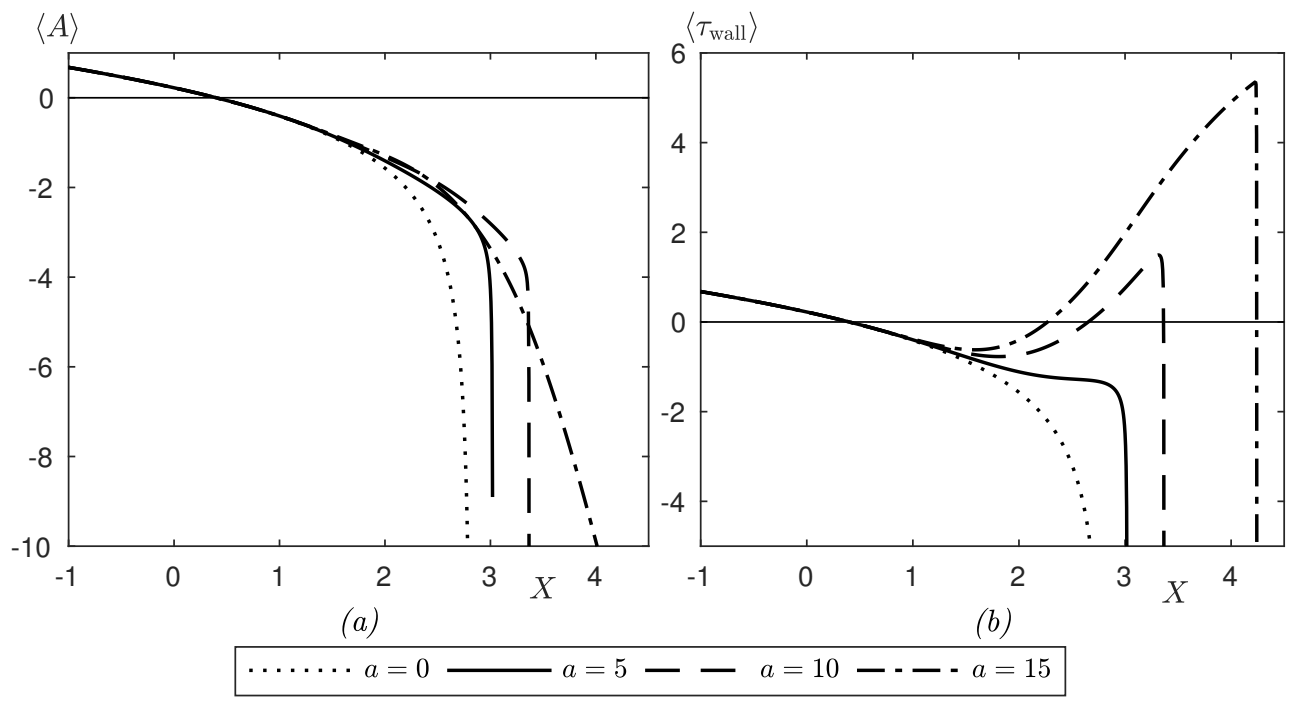

Figure 17. As in figure 14, but for a dynamic roughness oscillated with frequency $\omega=1.5$.

element, whether dynamic or static, in the region in which the Goldstein singularity was finally removed, allows one to shift both the point at which the time-averaged skin friction equals zero (the local separation point) and the later roughness region singularity (the breakaway separation point) further downstream.

The downstream (or upstream) movement of the local separation point has been seen to depend on the placement, width, height and, in the dynamic case, oscillation frequency of the roughness element. Despite the differences in configurations studied, the choice of parameters for the most downstream shift in the position of local separation is qualitatively similar to the required choice for the greatest increase in the critical parameter $\Gamma_{c}$, linked to the angle of attack, for Braun \& Kluwick (2004), above which no marginally separated flow solutions exist. The same is true for the most upstream shift of local separation point or decrease in $\Gamma_{c}$. We conclude, therefore, that a static roughness not only can have a beneficial impact in flow regimes other than that of Braun \& Kluwick, but that the parameter choices of the roughness are roughly the same. The use of larger roughness elements, centred downstream of the no-roughness singular point but with left endpoint upstream of it, are also able to move the breakaway separation point downstream and, in the case of a static roughness, significantly so. An extra forcing term upstream additional to the roughness shape itself could arise from the downstream behaviour at increased amplitude. It is interesting to note that static roughness elements, arranged in a spanwise-periodic manner at an appropriate chord location on an airfoil, may also be able to delay laminar-turbulent transition caused by crossflow instabilities (Saric et al. 2015). The study of negative static roughness elements indicates the formation firstly of separation bubbles as the depth is increased, followed by the sudden upstream advance of flow separation once the depth is above some critical value.

For the dynamic case, we found a similar impact of roughness height and oscillation frequency on the downstream shift of the singular point as that found by Huebsch et al. (2012) for effective flow control. Namely, that there is an inverse relationship between the two: higher frequencies allow one to use lower roughness heights to maintain the same streamwise delay in breakaway separation.

The governing equation for the displacement function (2.28) remains ill-posed in terms 
of standard time-marching, but if the time dependence is dealt with by expanding $A$ in Fourier modes, then the finite-time singularity can be avoided and a solution to the displacement obtained, for which the zeroth, steady Fourier mode is equal to the timeaveraged solution. We believe such an approach to be valid due to the fixed frequency forcing in our problem. Note that Braun \& Kluwick took their time dependence to be at an order higher than that of the steady obstacle shape and found that the resulting disturbance to the shear stress could be written as the multiple of two functions, $b$ and $u$, where $b$ depended only on the streamwise coordinate $X$ and $u$ was independent of it. Then $b$ was shown to be completely determined by the undisturbed flow over the obstacle, from which Braun \& Kluwick concluded that the response of the boundary layer in the streamwise direction was the same for all possible unsteady disturbances. This is not the case here, where the time-dependence is kept at leading order, and the oscillation of the roughness affects the flow behaviour as it develops along the roughness region.

It is worth also mentioning the impact of the roughness on the pressure. The overall pressure gradient in the roughness region is unfavourable, being equal to $h^{2} \mu>0$, and $P_{1}$ acts as an $O\left(h^{5 / 4}\right)$ correction to it. Thus a decreasing $P_{1}$ results in a favourable correction to the overall adverse pressure gradient and we have seen that both static and dynamic roughness are able to introduce a more favourable pressure gradient as the flow passes over the front of the roughness compared to where no roughness is present. Such a finding is consistent with previous experimental and direct computational findings concerning dynamic roughness: see, for example, figure 4 of Huebsch et al. (2012).

As an extension of the above comparison, an attempt to also tie in closely with both the previous analytical, experimental and numerical studies on dynamic roughness and the scenario of Braun \& Kluwick motivates our future work. The differences between their work and that presented in this paper include the simple linear form of the pressuredisplacement relation obtained here. In the absence of roughness elements, in which case the scaled skin friction equals the scaled displacement function, it states that a decrease in pressure results in a corresponding decrease in skin friction; this is contrary to what would be expected for flow over an airfoil, where favourable pressure gradients would increase the velocity of the airstream and thereby increase the skin friction.

It is of interest, therefore, to study the effect of the dynamic roughness examined here on marginally separated flow over an airfoil, where the time-dependence remains at leading order in the governing equations. Particularly, issues of importance concern first the ability of dynamic roughness elements to further increase the critical value of $\Gamma_{c}$, as Braun \& Kluwick found was possible for a steady roughness; second, the impact of the height and frequency compared to the pattern proposed by Huebsch et al.; third, how the skin friction and pressure behave; and fourth, how the stream functions respond. In addition, while 3D effects that may be significant in realistic configurations are absent in this investigation, it is felt that the $2 \mathrm{D}$ flow study sheds valuable light on the unexpected delicate phenomena present, including separating flow control, as described above.

The authors would like to thank Gregg Abate and Russell Cummings of the US Air Force Research Laboratory for very useful discussions over the course of this research. Support from AFOSR, under grant number FA9550-14-1-0240, is acknowledged gratefully. Helpful comments from the referees are also gratefully acknowledged. 


\section{REFERENCES}

Atik, H., Kim, C.-Y., Van Dommelen, L. L. \& Walker, J. D. A. 2005 Boundary-layer separation control on a thin airfoil using local suction. Journal of Fluid Mechanics 535, 415-443.

Braun, S. \& Kluwick, A. 2002 The effect of three-dimensional obstacles on marginally separated laminar boundary layer flows. Journal of Fluid Mechanics 460, 57-82.

Braun, S. \& Kluwick, A. 2004 Unsteady three-dimensional marginal separation caused by surface-mounted obstacles and/or local suction. Journal of Fluid Mechanics 514, 121-152.

Braun, S. \& Scheichl, S. 2014 On recent developments in marginal separation theory. Philosophical Transactions of the Royal Society A 372 (2020), 20130343.

Bushnell, D. M. \& Moore, K. J. 1991 Drag reduction in nature. Annual Review of Fluid Mechanics 23 (1), 65-79.

Chang, P. K. 1970 Separation of flow. Pergamon Press.

Dearing, S. S., Lambert, S. \& Morrison, J. F. 2007 Flow control with active dimples. Aeronautical Journal 111 (1125), 705-714.

Demauro, E. P., Dell'Orso, H., Zaremski, S., Leong, C. M. \& Amitay, M. 2015 Control of laminar separation bubble on NACA 0009 airfoil using electroactive polymers. AIAA Journal 53 (8), 2270-2279.

Fish, F. E. \& Lauder, G. V. 2006 Passive and active flow control by swimming fishes and mammals. Annual Review of Fluid Mechanics 38, 193-224.

Goldstein, S. 1948 On laminar boundary-layer flow near a position of separation. The Quarterly Journal of Mechanics and Applied Mathematics 1 (1), 43-69.

Grager, T., Rothmayer, A. P., Huebsch, W. W. \& Hu, H. 2012 Low Reynolds number stall suppression with dynamic roughness. In 6th AIAA Flow Control Conference, AIAA Paper 2012-2681, pp. 25-28. American Institute of Aeronautics and Astronautics.

Gad-el HaK, M. 2000 Flow Control: Passive, Active and Reactive Flow Management. Cambridge University Press.

Hsiao, C.-T. \& Pauley, L. L. 1994 Comparison of the triple-deck theory, interactive boundary layer method, and Navier-Stokes computation for marginal separation. Trans. ASME J. Fluids Engng 116, 22-28.

Huebsch, W. W. 2006 Two-dimensional simulation of dynamic surface roughness for aerodynamic flow control. Journal of Aircraft 43 (2), 353-362.

Huebsch, W. W., Gall, P. D., Hamburg, S. D. \& Rothmayer, A. P. 2012 Dynamic roughness as a means of leading-edge separation flow control. Journal of Aircraft 49 (1), $108-115$.

Lissaman, P. B. 1983 Low-Reynolds-number airfoils. Annual Review of Fluid Mechanics 15 (1), 223-239.

Modi, V. J. 1997 Moving surface boundary-layer control: A review. Journal of Fluids and Structures 11, 627-663.

Prandth, L. 1904 Uber flussigkeits bewegung bei sehr kleiner reibung. Verhaldlg III Int. Math. Kong pp. 484-491.

Pruessner, L. \& Smith, F. T. 2015 Enhanced effects from tiny flexible in-wall blips and shear flow. Journal of Fluid Mechanics 772, 16-41.

Rothmayer, A. P. \& Huebsch, W. W. 2011 On the modification of laminar boundary layers using unsteady surface actuation. In 6th AIAA Theoretical Fluid Mechanics Conference, AIAA Paper 2011-4016. American Institute of Aeronautics and Astronautics.

Rothmayer, A. P. \& Huebsch, W. W. 2012 Flow about large unsteady two-dimensional humps in a boundary layer. In 42nd AIAA Fluid Dynamics Conference and Exhibit, AIAA Paper 2012-2949, p. 2949.

Ruban, A.I. 1981 Singular solution of boundary layer equations which can be extended continuously through the point of zero surface friction. Fluid Dynamics 16 (6), 835-843.

Ruban, A. I. 1982 Asymptotic theory of short separation regions on the leading edge of a slender airfoil. Fluid Dynamics 17 (1), 33-41.

Saric, W., West, D., Tufts, M. \& Reed, H. 2015 Flight test experiments on discrete roughness element technologry for laminar flow control. AIAA Paper 539, 2015.

Schlichting, H. \& Gersten, K. 2000 Boundary Layer Theory. Springer. 
Smith, F. T. 1976a Flow through constricted or dilated pipes and channels: Part 1. The Quarterly Journal of Mechanics and Applied Mathematics 29 (3), 343-364.

Smith, F. T. $1976 b$ Flow through constricted or dilated pipes and channels: Part 2. The Quarterly Journal of Mechanics and Applied Mathematics 29 (3), 365-376.

Smith, F. T. 1982 Concerning dynamic stall. Aeronautical Quarterly 33, 331-352.

Smith, F. T., Brighton, P. W. M., Jackson, P. S. \& Hunt, J. C. R. 1981 On boundarylayer flow past two-dimensional obstacles. Journal of Fluid Mechanics 113, 123-152.

Smith, F. T. \& Daniels, P. G. 1981 Removal of Goldstein's singularity at separation, in flow past obstacles in wall layers. Journal of Fluid Mechanics 110, 1-37.

Stewartson, K. 1970 Is the singularity at separation removable? Journal of Fluid Mechanics $44(2), 347-364$.

Stewartson, K., Smith, F. T. \& Kaups, K. 1982 Marginal separation. Studies in Applied Mathematics 67, 45-61.

Sychev, V. V., Ruban, A. I., Sychev, V. V. \& Korolev, G. L. 1998 Asymptotic theory of separated flows. Cambridge University Press.

TANi, I. 1969 Boundary-layer transition. Annual Review of Fluid Mechanics 1 (1), 169-196.

Zametaev, V. B. 1986 Existence and nonuniqueness of local separation zones in viscous jets. Fluid Dynamics 21 (1), 31-38. 